\title{
The staircase method: integrals for periodic reductions of integrable lattice equations
}

\author{
Peter H. van der Kamp, G.R.W. Quispel \\ email: P.vanderKamp@LaTrobe.edu.au \\ Department of Mathematics and Statistics \\ La Trobe University \\ Victoria 3086, Australia
}

date: November 14, 2018

PACS: 02.30.Ik, 05.50.Ik, 02.60.Dc, 05.45.Ra, 45.90.+t, 89.75.Fb

Keywords: Lattice equation, Mapping, Correspondence, Integrable, Staircase method, Periodic reduction, Reduction of order, Integral, $k$-Symmetry, Quad-graph

\begin{abstract}
We show, in full generality, that the staircase method [27, 29] provides integrals for mappings, and correspondences, obtained as traveling wave reductions of (systems of) integrable partial difference equations. We apply the staircase method to a variety of equations, including the KortewegDe Vries equation, the five-point Bruschi-Calogero-Droghei equation, the QD-algorithm, and the Boussinesq system. We show that, in all these cases, if the staircase method provides $r$ integrals for an $n$-dimensional mapping, with $2 r<n$, then one can introduce $q \leq 2 r$ variables, which reduce the dimension of the mapping from $n$ to $q$. These dimension-reducing variables are obtained as joint invariants of $k$-symmetries of the mappings. Our results support the idea that often the staircase method provides sufficiently many integrals for the periodic reductions of integrable lattice equations to be completely integrable. We also study reductions on other quad-graphs than the regular $\mathbb{Z}^{2}$ lattice, and we prove linear growth of the multi-valuedness of iterates of high-dimensional correspondences obtained as reductions of the QD-algorithm.
\end{abstract}




\section{Introduction}

The field of integrable partial difference equations emerged in the late nineteen seventies, early eighties $[1,11,17,23,30]$. An important, and well-studied, class of partial difference equations is the class of (scalar) equations that are defined on the elementary squares of a lattice. An example of an integrable equation in this class is the lattice potential Korteweg-de Vries (pKdV) equation

$$
\left(u_{l, m}-u_{l+1, m+1}\right)\left(u_{l+1, m}-u_{l, m+1}\right)=\alpha,
$$

in which the linear terms are transformed away. Such equations are part of a multi-dimensional family of mutually consistent partial difference equations $[20,24]$. A classification with respect to multi-dimensional consistency has been achieved recently $[2,3]$.

For lattice equations on the square, an initial value problem can be posed on a so called staircase: a connected path which is nondecreasing, or nonincreasing. In [27] initial values are given at lattice points $u_{l, l}$ and $u_{l+1, l}$ which satisfy the periodicity $u_{l, m}=u_{l+p, m+p}$. By doing so, the partial difference equation $(P \Delta E)$ reduces to a multi-dimensional mapping. The authors of [27] used the linear spectral problem (Lax pair) of the lattice pKdV equation to derive a set of polynomial invariants for this mapping. They constructed a so called monodromy matrix, which is an ordered product of Lax matrices along the staircase over a one-period distance. This method, nowadays known as the staircase method, is an important tool in proving complete integrability in the sense of Liouville-Arnold. Here, a $2 n$-dimensional mapping is said to be completely integrable if it admits $n$ functionally independent integrals in involution with respect to a symplectic form $[7,42]$. Thus, the number of integrals should be at least equal to half the dimension of the mapping.

In $[10,21]$ the authors established the involutivity of the integrals for the mappings they introduced in [27]. Similar results have been obtained for mappings derived from the lattice Gel'fand-Dikii hierarchy [22] and for reductions of the time-discrete versions of the Bogoyavlensky equations [26]. In [29] more general staircases were given, corresponding to the s-periodicity condition: ${ }^{1}$

$$
u_{l, m}=u_{l+s_{1}, m+s_{2}}
$$

where $s_{1},-s_{2} \in \mathbb{N}^{+}$are relatively prime integers. The authors also suggested considering general $s_{1},-s_{2} \in \mathbb{N}^{+}$, see the third concluding remark in that paper. In recent work $[38,33]$ we have provided a unified picture for s-periodic reductions, with nonzero $\mathbf{s}=\left(s_{1}, s_{2}\right) \in \mathbb{Z} \times \mathbb{Z}$. In [33] it was shown how, under periodicity condition (2), any lattice equation $f\left(u_{l, m}, \cdots\right)=0$ reduces to a system of $r$ ordinary difference equations $f\left(v_{n}^{p}, \cdots\right)=0, p=0, \ldots, r-1$, where $r$ is the greatest common divisor of $s_{1}$ and $s_{2}$. Also it was proved that the monodromy matrix, denoted $\mathcal{L}$, is one of the Lax matrices for the reduction, that is, there exists a matrix $\mathcal{M}$ such that for (periodic) solutions of the system

\footnotetext{
${ }^{1}$ Our notation differs from the one used in [29], where $s_{1}=z_{2}$ and $s_{2}=-z_{1}$.
} 
the following holds,

$$
\mathcal{L}_{n} \mathcal{M}_{n}=\mathcal{M}_{n} \mathcal{L}_{n+1}
$$

In [38] a geometric description of s-reduction has been given. It was shown that for all $\mathbf{s}$ there exists a well-posed, or nearly well-posed, s-periodic initial value problem, for any given scalar lattice equation on some arbitrary stencil of lattice points. We expect something similar to hold for systems of lattice equations, cf. [34]. Combining the two results; given the existence of a (nontrivial) periodic solution, after multiplying equation (3) by $\mathcal{M}_{n}^{-1}$, we may conclude that the trace of $\mathcal{L}$ is an invariant of the mapping $n \mapsto n+1$. In section 2 we provide a direct proof, in the spirit of the original work [29], that the staircase method applies to any given system of lattice equations, if a Lax-pair and a (nearly) well-posed periodic initial value problem are known.

Note, for equations on a square the monodromy matrix is defined on the staircase, and the initial conditions are given at all points of the same staircase. However, for equations on other stencils, and for systems of equations, the monodromy matrix is still given on the staircase, but the initial conditions no longer correspond to the points on the staircase.

The trace of the monodromy matrix $\mathcal{L}$ depends on a spectral parameter, arising from the Lax representation of the $\mathrm{P} \Delta \mathrm{E}$. By expanding in this parameter we obtain a number of integrals. In relation to establishing the complete integrability of a mapping (or correspondence) obtained by periodic reduction a first question to ask is: does the staircase method yield sufficiently many functionally independent integrals?

For the reductions we perform in section 3.1.1, of the Bruschi-CalogeroDroghei equation [6]

$$
\left(u_{l, m}-u_{l, m-1}\right)\left(u_{l, m}-u_{l-1, m-1}\right)=\left(u_{l, m}-u_{l, m+1}\right)\left(u_{l, m}-u_{l+1, m+1}\right),
$$

the number of integrals is exactly half the dimension of the mapping. For the one-parameter families of reductions we perform in section 3.1.2, of the QDsystem [25]

$$
e_{l, m+1}+q_{l+1, m+1}=q_{l+1, m}+e_{l+1, m}, \quad e_{l, m+1} q_{l, m+1}=q_{l+1, m} e_{l, m},
$$

we have verified that the number of integrals is one more than half the dimension of the mapping. In other cases, there are fewer integrals than half the dimension of the mapping obtained by periodic reduction. For example, performing periodic reductions of the lattice pKdV equation (1) we find $(2 n+1)$-dimensional mappings and $(2 n+2)$-dimensional mappings, for which the staircase method provides only $n$ integrals, see section 4.2.1. It turns out that when the dimension of the mapping is $2 n+1$ it can be dimensionally reduced by 1 , whereas when the dimension is $2 n+2$ it can be reduced by 3 . All $n$ integrals survive the dimensional reduction and we can conclude that the dimensionally reduced mappings posses sufficiently many integrals for complete integrability.

To distinguish the two kinds of reductions we say s-reduction for a periodic reduction of a lattice equation with period $\mathbf{s} \in \mathbb{Z} \times \mathbb{Z}$ to a multi-dimensional 
mapping, and we say d-reduction for a reduction of order which reduces the dimension of a mapping by $d \in \mathbb{N}$.

In section 4.3 we show how to pose s-periodic initial value problems for the Boussinesq system $[22,35]$

$$
\begin{aligned}
w_{l+1, m}+v_{l, m} & =u_{l, m} u_{l+1, m}, \\
w_{l, m+1}+v_{l, m} & =u_{l, m} u_{l, m+1}, \\
w_{l, m}+v_{l+1, m+1} & =u_{l, m} u_{l+1, m+1}+\frac{\gamma}{u_{l+1, m}-u_{l, m+1}} .
\end{aligned}
$$

Performing s-reduction with $\mathbf{s}=(n-1,1)$ we get a $2 n$ dimensional mapping. For these mappings we verified, for all $n \leq 17$, that the staircase method provides $n-1$ integrals unless 3 divides $n$ in which case it provides only $n-3$ functionally independent integrals. We show, for all $n$, that the mapping can be 6 -reduced if 3 divides $n$, and that the mapping can be 2-reduced otherwise.

These examples suggest that if the staircase method provides $r$ integrals for an $n$-dimensional mapping, with $2 r<n$, then the mapping can be $d$-reduced, with $d \geq n-2 r$. However, we do not claim the above statement is true in general; in examples given in [34] the staircase method gives integrals of the form $J J^{\prime}$ where $J$ is a 2-integral, and it does not produce the integral $J+J^{\prime}$. Recall, a function $J$ is an $k$-integral, or $k$-symmetry, of a mapping if it is an integral, or symmetry, of the $k^{\text {th }}$ power of that mapping [13]. If one has one $k$-integral, then one can construct $k$ of them, or, even better, $k$ integrals. For example, it is easy to see that $J^{\prime \prime}=J$ implies that both $J J^{\prime}$ and $J+J^{\prime}$ are integrals. In all cases considered in this paper, the $d$-reduction is performed by introducing $n-d$ new variables, which can be obtained as the joint invariants of symmetries, or $k$-symmetries, of the mapping, which in turn are obtained from point-symmetries of the partial difference equation. This will be explained in section 4 .

Recently, in [4], a geometric criterion was given for the well-posedness of initial value problems on quad-graphs. In section 5 we will show that for 'regular' quad-graphs, those that permit periodic solutions, the staircase method can be applied. We study reductions of equation $H 3_{\delta=0}$ from [2], which on a $\mathbb{Z}^{2}$-lattice would look like

$$
p\left(u_{l, m} u_{l+1, m}+u_{l, m+1} u_{l+1, m+1}\right)=q\left(u_{l, m} u_{l, m+1}+u_{l+1, m} u_{l+1, m+1}\right) .
$$

We will consider two different quad-graphs, namely Figure 9d and 9e in [4]. These quad-graphs carry more lattice parameters than the standard $\mathbb{Z}^{2}$ lattice, and these parameters do all appear in the reduced mapping. In the second case the lattice parameters are interchanged by the shift on the quad-graph, and we find the reduction to be an alternating mapping, cf. [28].

For certain s-reductions the periodic solutions are given by multi-valued mappings, or correspondences, see [38]. The staircase method applies equally well in such cases, see section 4.3.1, where we perform (3,0)-reduction for the pKdV equation, and section 3.2.1, where we $(n, 0)$-reduce the QD-system. Here, 
another question arises: what is the multi-valuedness of the iterates of the correspondence?

In general, the number of image points of the $n$th iterate of an $m$-valued correspondence would be $m^{n}$. However, it has been shown that for completely integrable correspondences the number of images under the iterates grows polynomially, rather than exponentially [41]. In section 6 we show that for the correspondences obtained in sections 4.3.1 and 3.2.1 the multi-valuedness of their $n$th iterate is $n+1$ and $2 n$, respectively.

\section{The staircase method, general theory}

Let $\mathbf{u}$ be a multi-component field on the square lattice $\mathbb{Z}^{2}$ and let $\mathbf{f}[\mathbf{u}]$ be a multi-component function of $\mathbf{u}$ and finitely many shifts of $\mathbf{u}$. We call a lattice equation $\mathbf{f}[\mathbf{u}]=0$ integrable if it arises as the compatibility condition of two linear equations $\widetilde{\psi}=L \psi$ and $\widehat{\psi}=M \psi$. Here, $\sim$ denotes the horizontal shift $l \mapsto l+1$ and ${ }^{\wedge}$ denotes the vertical shift $m \mapsto m+1$. Thus we have

$$
\widehat{L} M \equiv \widetilde{M} L \bmod \mathbf{f},
$$

which is called the Lax-equation, or zero-curvature condition. The matrices $L$ and $M$ are called Lax matrices.

As pointed out by Calogero and Nucci [9] in the continuum case, see also [12], the mere existence of a Lax pair is not sufficient for integrability, the Lax pair has to be a good Lax pair. In the discrete setting one has to be equally careful, see chapter 6 in the thesis of Mike Hay [14]. From this point of view one could argue that the staircase method tests whether a Lax pair is 'good'. The Lax pair would be called good (and hence the lattice equation integrable), if it can be used to produce a sufficient number of integrals for periodic reductions.

We say that a lattice equation $\mathbf{f}[\mathbf{u}]=0$ admits a well-posed initial value problem if from a set of generic initial points a solution can be constructed in a unique way. An initial value problem is called nearly-well-posed if from a set of generic initial points solutions can be constructed, which can take only finitely many values at each lattice point. We consider periodic initial value problems. In the first case the solution is obtained by iterating a (finite dimensional) mapping. In the second case the solutions are obtained by iterating a correspondence. We note that if the initial value problem is well-posed the periodicity of the solution is implied by the periodicity of the initial values, whereas when in the case of nearly well-posedness the periodicity of the solutions is imposed.

Theorem 1 Let $\mathbf{a}, \mathbf{s}$ be elements of $\mathbb{Z} \times \mathbb{Z}$. Suppose an integrable equation $\mathbf{f}[\mathbf{u}]=0$ allows a $\mathbf{s - p e r i o d i c ~ i n i t i a l ~ v a l u e ~ p r o b l e m ~ w h i c h ~ i s ~ w e l l - p o s e d , ~ o r ~ n e a r l y - ~}$ well-posed. Then, with $\mathcal{L}$ being an inversely ordered product of Lax matrices over a connected path, e.g. a staircase, from $\mathbf{a}$ to $\mathbf{a}+\mathbf{s}$, the trace of $\mathcal{L}^{i}$ is invariant under any shift on the lattice, $\forall i \in \mathbb{N}$.

Proof: Let $\mathbf{a}, \mathbf{b}$ be two points on the lattice. Define $\mathcal{L}_{\mathbf{a}, \mathbf{b}}$ to be the inversely ordered product of Lax matrices along a connected path from $\mathbf{a}$ to $\mathbf{b}$. We have to 
show that $\mathcal{L}_{\mathbf{a}, \mathbf{b}}$ does not depend on the path from a to $\mathbf{b}$. This follows from the fact that every square can be passed in two ways: if $L, M$ are the Lax matrices at $\mathbf{a} \in \mathbb{Z}^{2}$ (and $\widetilde{L}$ is a Lax matrix at $\widetilde{\mathbf{a}}=\mathbf{a}+(1,0)$ ), then from (4) it follows that $\widehat{L} M=\widetilde{M} L=\mathcal{L}_{\mathbf{a}, \widehat{\widetilde{\mathbf{a}}}}$ is well-defined for solutions of $\mathbf{f}[\mathbf{u}]=0$. We have

$$
\mathcal{L}_{\widetilde{\mathbf{a}}, \widetilde{\mathbf{b}}}=\mathcal{L}_{\mathbf{b}, \widetilde{\mathbf{b}}} \mathcal{L}_{\mathbf{a}, \mathbf{b}} \mathcal{L}_{\widetilde{\mathbf{a}}, \mathbf{a}}=\mathcal{L}_{\mathbf{b}, \widetilde{\mathbf{b}}} \mathcal{L}_{\mathbf{a}, \mathbf{b}} \mathcal{L}_{\mathbf{a}, \widetilde{\mathbf{a}}}^{-1}
$$

Because the initial value problem is well-posed, or nearly-well-posed, there exists an s-periodic solution. Now let $\mathbf{b}=\mathbf{a}+i \mathbf{s}(i \in \mathbb{N})$, so that the value of the solution at $\mathbf{a}$ and $\mathbf{b}$ coincide. Then $\mathcal{L}_{\mathbf{a}, \mathbf{b}}=\mathcal{L}^{i}$. Also, $\mathcal{L}_{\mathbf{b}, \widetilde{\mathbf{b}}}=\mathcal{L}_{\mathbf{a}, \widetilde{\mathbf{a}}}$. If we denote $I=\operatorname{Tr}\left(\mathcal{L}^{i}\right)$, it is clear that we have $\widetilde{I}=I$ and, similarly $\widehat{I}=I$.

The mapping, or correspondence, which generates the s-periodic solution is defined by updating a set of initial values through a shift on the lattice. Therefore, an invariant for it is given by the trace of (an integer power of) $\mathcal{L}$. If the Lax-matrices depend on a spectral parameter, say $k$, one can expand the trace $\operatorname{Tr}\left(\mathcal{L}^{i}\right)$ in powers of $k$. Each coefficient then provides an integral for the mapping, or for the correspondence. However, these integrals are not all functionally independent.

By the Cayley-Hamilton theorem any matrix $\mathcal{L}$ satisfies its own characteristic equation $P(\lambda)=\operatorname{Det}(\lambda I-\mathcal{L})$, i.e. we have $P(\mathcal{L})=0$. Therefore, given that $\mathcal{L}$ is a $n \times n$ matrix, it suffices to consider traces of $\mathcal{L}^{i}$, with $i \leq n$. Even better, there are certain combinations of $\operatorname{Tr}\left(\mathcal{L}^{i}\right)$, which, generally, yield a nicer basis of functionally independent integrals. These are provided by the coefficients in $P(\lambda)=0$. For example, if $n=2$ we have

$$
P(\lambda)=\lambda^{2}-\lambda \operatorname{Tr}(\mathcal{L})+\left(\operatorname{Tr}(\mathcal{L})^{2}-\operatorname{Tr}\left(\mathcal{L}^{2}\right)\right) / 2 .
$$

Note that the coefficient of $\lambda^{0}$ coincides with the determinant of $\mathcal{L}$.

For general $n$, the coefficients can be obtained using Newton's identities

$$
n e_{n}=\sum_{i=1}^{n}(-1)^{i-1} e_{n-i} p_{i}
$$

where the power sums $p_{k}$ are given by $p_{k}=x_{1}^{k}+x_{2}^{k}+\cdots+x_{q}^{k}$ and the elementary symmetric polynomials $e_{k}$ are given by

$$
e_{k}=\sum_{1 \leq i_{1}} \sum_{i_{1}<i_{2}} \ldots \sum_{i_{k-1}<i_{k}} \sum_{i_{k} \leq q} \prod_{j=1}^{k} x_{i_{j}},
$$

and appear as coefficients in the (Vieta) expansion

$$
\prod_{i=1}^{q}\left(\lambda-x_{i}\right)=\sum_{i=0}^{q}(-1)^{i} e_{i} \lambda^{q-i} .
$$

If we denote the $q$ eigenvalues of the matrix $\mathcal{L}$ by $x_{i}$, the characteristic polynomial equals the left hand side of equation (7). Using Newton's identities (6) the 
right hand side can be expanded recursively in terms of $p_{k}=\operatorname{Tr}\left(\mathcal{L}^{k}\right)$. Taking $k=1,2$ in (6) we find familiar coefficients $e_{1}=p_{1}, e_{2}=\left(p_{1}^{2}-p_{2}\right) / 2$, see equation (5). Taking $k=3,4$ Newton's identities yield $e_{3}=\left(p_{1}^{3}-3 p_{1} p_{2}+2 p_{3}\right) / 6$, and $e_{4}=\left(p_{1}^{4}-6 p_{1}^{2} p_{2}+3 p_{2}^{2}+8 p_{1} p_{3}-6 p_{4}\right) / 24$.

For scalar equations that are defined on elementary squares, initial values are given on staircases. So the dimension of the initial value problem is $\left|s_{1}\right|+\left|s_{2}\right|$. A so-called standard staircase, cf. $[30,33,38]$ gives rise to a particularly simple mapping. In fact, any mapping, defined by a shift on the lattice, is (equivalent to) a certain iterate of this basic one.

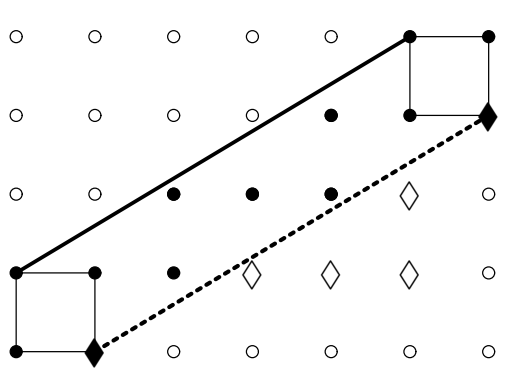

Figure 1: (5,3)-periodic initial value problem for equations defined on elementary squares
To illustrate this, we have presented the standard (5,3)-staircase in Figure 1. The standard staircase is the path through the points between the two lines, the black dots. They are the points where initial values are given. The standard mapping will be the shift $u_{l, m} \mapsto$ $u_{l+2, m+1}$. Note that by this shift almost all black dots are shifted to another black dot. The one black dot which is closest to the dotted line, is shifted to the black diamond, whose value can be calculated using the equation on the square.

We can also see that the mapping defined by the shift $u_{l, m} \mapsto u_{l+1, m}$ is given as the third power of the standard one. To evaluate the monodromy matrix $\mathcal{L}$, one would take the product of matrices along the same staircase on which the initial values are given. Since for this type of equations the matrices $L, M$ depend on $(u, \widetilde{u}),(u, \widehat{u})$, respectively, the matrix $\mathcal{L}$ is then automatically expressed in terms of the initial values. We note that one can just as well take the product over any other one-period long path. For example, assuming that $\mathbf{s} \in \mathbb{N} \times \mathbb{N}$, one could consider the product

$$
\mathcal{L}(l, m)=\prod_{j=0}^{\substack{s_{2}-1 \\ s^{\prime}}} M_{l+s_{1}, m+j} \prod_{i=0}^{\substack{s_{1}-1 \\ L}} L_{l+i, m} .
$$

Then, one first has to calculate the points on the corresponding path to be able to evaluate $\mathcal{L}$. In the example given in Figure 1, we would need to calculate the values of the field at the white diamonds, which amounts to iterating the mapping 8 times.

For equations, or systems, that are not defined on elementary squares the initial value problem does, in general, not lie on a staircase. Depending on the type of stencil and on the particular periodicity condition, there could either be more, or less than $\left|s_{1}\right|+\left|s_{2}\right|$ initial values. In [38] it was shown how to write down, for a given scalar equation on an arbitrary stencil, a piece-wise linear expression (as a function of $\mathbf{s}$ ) for the dimension of an $\mathbf{s}$-periodic initial value problem. 
Note that the monodromy matrix is still a product of $\left|s_{1}\right|+\left|s_{2}\right|$ matrices (if the product is taken over a staircase, which is the sensible thing to do). Also note that, in general, the Lax matrices depend on $\mathbf{u}$ and a number of shifts of $\mathbf{u}$. Therefore, for certain choices of $\mathbf{s}$, one needs to determine a number of points, by iterations of the mapping, or correspondence, in order to evaluate the monodromy matrix in terms of the initial values. It might be possible to avoid this by using the equation to change the $[\mathbf{u}]$-dependence of the Lax-matrices. However, that would have to be adjusted to the particular choice of $\mathbf{s}$.

\section{The staircase method, applications}

In this section we will apply the staircase method to a variety of different equations and different reductions. Firstly, we consider a 5-point equation, i.e. an equation which is not defined on the square. Second, we calculate integrals for reductions of a 2-component system of equations. We first perform reductions which give rise to mappings and then we also perform reductions that yield correspondences.

\subsection{Mappings with a sufficient number of integrals}

\subsubsection{The Bruschi-Calogero-Droghei equation}

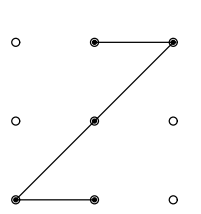

Figure 2

In [6] one finds the five-point equation $E(u, \widehat{u}, \underset{\sim}{u}, \widetilde{\widehat{u}}, \underset{\sim}{u})=0$, where

$$
E=(u-\underset{\sim}{u})(u-\underset{\sim}{u})-(u-\widehat{u})(u-\widetilde{\widehat{u}}),
$$

see $\left[6\right.$, Equation (4a)] in which we have set $\alpha^{(\nu)}=0$. The equation is defined on the stencil given in Figure 2.

The two recursive formulas [6, Equations (1a),(6)], with coefficients [6, Equations (5),(7)], yield the following Lax-pair

$$
\begin{aligned}
L(u, \widetilde{u}, \underset{\sim}{u} \underset{\sim}{u})=\left(\begin{array}{cc}
k+\widetilde{u}-u & (u-\underset{\sim}{u})(u-\underset{\sim}{u}) \\
1 & 0
\end{array}\right), \\
M(u, \widehat{u}, \underset{\sim}{u})=\left(\begin{array}{cc}
1 & (\widehat{u}-u) \\
(\widehat{u}-\underset{\sim}{u})^{-1} & 1-(k+u-\underset{\sim}{u})(\widehat{u}-\underset{\sim}{u})^{-1}
\end{array}\right) .
\end{aligned}
$$

Here we have denoted the spectral parameter ( $x$ in [6]) by $k$, which we do throughout this paper. The method laid out in [38] tells us how to pose welldefined s-periodic initial value problems for this equation. This can be done for all $\mathbf{s}=\left(s_{1}, s_{2}\right)$ such that $s_{2}\left(s_{2}-2 s_{1}\right) \neq 0$. The dimension of the periodic solutions is given by the following piecewise-linear function $2 \max \left\{\left|s_{2}-s_{1}\right|,\left|s_{1}\right|\right\}$. We apply the staircase method to a few reductions, in the different regions distinguished by this function, see [38, Figure 10]. 


\section{$(0,3)$-reduction}

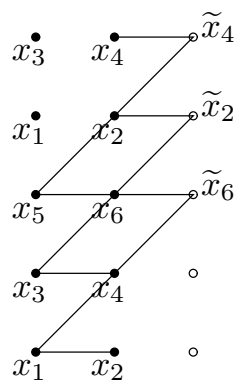

Figure 3

We assigning initial values as in Figure 3. They are indicated by the black dots. We update them using the right-shift, the values of $\widetilde{x_{2}}, \widetilde{x_{4}}, \widetilde{x_{6}}$ can be determined using the equations indicated by the zig-zags. We get a sixdimensional mapping

$$
\left(\begin{array}{ll}
x_{1} & x_{2} \\
x_{3} & x_{4} \\
x_{5} & x_{6}
\end{array}\right) \mapsto\left(\begin{array}{ll}
x_{2} & \frac{x_{2} x_{6}+x_{3} x_{4}-x_{3} x_{6}-x_{4} x_{6}}{x_{2}-x_{6}} \\
x_{4} & \frac{x_{2} x_{5}+x_{2} x_{6}-x_{2} x_{4}-x_{5} x_{6}}{x_{2}-x_{4}} \\
x_{6} & \frac{x_{1} x_{4}+x_{2} x_{4}-x_{1} x_{2}-x_{4} x_{6}}{x_{4}-x_{6}}
\end{array}\right) .
$$

The monodromy matrix, which we take from $x_{2}$ upwards to $x_{2}$, is $\mathcal{L}=M\left(x_{6}, x_{2}, x_{5}\right) M\left(x_{4}, x_{6}, x_{3}\right) M\left(x_{2}, x_{4}, x_{1}\right)$.

Three functionally independent integrals for this mapping can be obtained from the coefficients in its characteristic polynomial (5). They are $\left(x_{1}-x_{4}\right)\left(x_{2}-\right.$ $\left.x_{5}\right)\left(x_{3}-x_{6}\right),\left(x_{1}-x_{6}\right)\left(x_{2}-x_{3}\right)\left(x_{4}-x_{5}\right)$, and $\left(x_{1}-x_{5}\right)\left(x_{2}-x_{4}\right)+\left(x_{3}-x_{5}\right)\left(x_{4}-x_{6}\right)$.

\section{(1,3)-reduction}

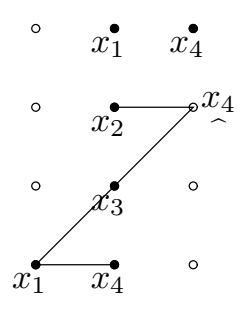

Figure 4
Assigning initial values as in Figure 4, and updating them using the down-shift, we get a four-dimensional mapping

$$
\left(x_{1}, x_{2}, x_{3}, x_{4}\right) \mapsto\left(x_{2}, x_{3}, x_{4}, \frac{x_{1} x_{4}+x_{2} x_{3}-x_{1} x_{3}-x_{3} x_{4}}{x_{2}-x_{3}}\right) .
$$

In this case we have to first calculate a few values of the field at points close to the staircase in order to evaluate the monodromy matrix. We calculate $\widehat{x_{1}}$ by solving $E\left(x_{2}, x_{1}, x_{3}, x_{4}, \widehat{x_{1}}\right)=0$, and we find $\widehat{\widehat{x_{1}}}$ from $E\left(x_{1}, \widehat{x_{1}}, x_{2}, x_{3}, \widehat{x_{1}}\right)=0$.

Two functionally independent integrals are obtained from the coefficients in (5) with $\mathcal{L}=M\left(x_{2}, x_{1}, \widehat{\widehat{x}}_{1}\right) M\left(x_{3}, x_{2}, \widehat{x}_{1}\right) M\left(x_{4}, x_{3}, x_{1}\right) L\left(x_{1}, x_{4}, x_{2}, \widehat{\widehat{x}}_{1}\right)$. They are $J_{1}=\left(x_{1}-x_{2}\right)\left(x_{3}-x_{4}\right)$, and $J_{2}=\left(x_{1}-x_{3}\right)\left(x_{2}-x_{4}\right) /\left(x_{2}-x_{3}\right)$.

\section{$(2,3)$-reduction}

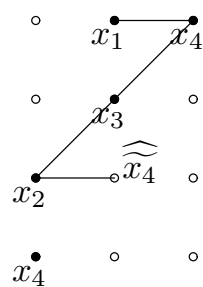

Figure 5
We assign initial values as in Figure 5 and update them using the diagonal shift $u \mapsto \widehat{\widetilde{u}}$. The values $\widetilde{x}_{2}$, which equals $\widehat{\bar{x}_{4}}$, and $\widetilde{x}_{3}$ are determined by $E\left(x_{3}, x_{1}, \widetilde{x}_{2}, x_{4}, x_{2}\right)=0$, and $E\left(x_{4}, x_{2}, \widetilde{x}_{3}, \widetilde{x}_{2}, x_{3}\right)=0$, successively. We find the same four-dimensional mapping as in the previous case. The monodromy matrix $M\left(x_{4}, x_{2}, x_{1}\right) M\left(\widetilde{x}_{3}, x_{4}, x_{3}\right) L\left(x_{3}, \widetilde{x}_{3}, \widetilde{x}_{2}, x_{2}\right) M\left(\widetilde{x}_{2}, x_{3}, x_{2}\right)$. $L\left(x_{2}, \widetilde{x}_{2}, x_{4}, x_{1}\right)$ yields the same integrals $J_{1}, J_{2}$. 


\section{$(2,-1)$-reduction}

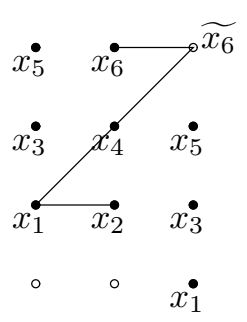

Figure 6

Assigning initial values as in Figure 6, and updating them using the right-shift, we get a six-dimensional mapping

$$
\begin{aligned}
x_{i} & \mapsto x_{i+1}, \quad i \in\{1,2, \ldots 5\}, \\
x_{6} & \mapsto \frac{x_{1} x_{4}+x_{2} x_{4}-x_{1} x_{2}-x_{4} x_{6}}{x_{4}-x_{6}} .
\end{aligned}
$$

The trace of the monodromy matrix $M^{-1}\left(x_{4}, x_{6}, x_{3}\right) L\left(x_{5}, x_{6}, x_{3}, x_{2}\right) L\left(x_{4}, x_{5}, x_{2}, x_{1}\right) \quad$ yields two functionally independent integrals

These are $\left(x_{1}-x_{6}\right)\left(x_{2}-x_{4}\right)\left(x_{3}-x_{5}\right)$ and $\left(x_{2}-x_{6}\right)\left(x_{3}-x_{5}\right)+\left(x_{1}-x_{4}\right)\left(x_{2}-\right.$ $\left.x_{4}\right)+\left(x_{3}-x_{4}\right)\left(x_{4}-x_{5}\right.$. A third functionally independent integral, $\left(x_{1}-x_{4}\right)\left(x_{2}-\right.$ $\left.x_{4}\right)\left(x_{2}-x_{5}\right)\left(x_{3}-x_{5}\right)\left(x_{3}-x_{6}\right)$, is obtained by taking the determinant of the monodromy matrix. In the previous cases, the determinant does not provide a functionally independent integral.

\subsubsection{The QD-algorithm}

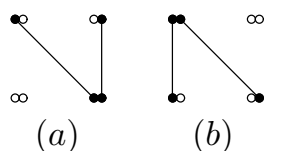

Figure 7:

QD-type system

The quotient-difference (QD) algorithm,

$$
\begin{aligned}
\widehat{e}+\widehat{\widetilde{q}} & =\widetilde{q}+\widetilde{e}, \\
\widehat{e} \widehat{q} & =e \widetilde{q},
\end{aligned}
$$

is used to construct continued fractions whose convergents form ordered sequences in a normal Padé table [8], and to find the zeros of a polynomial [15].

It is also called the time-discrete Toda molecule [19]. It is an integrable twocomponent equation defined on the stencils depicted in Figure 7, where we associate two values, $e$ on the left and and $q$ on the right, to every point on the lattice.

A Lax-pair for the QD-algorithm can be obtained from relations between so called higher adjacent orthogonal polynomials [5], cf. [25, equations $(3,4)]$

$$
k \widehat{P}=\widetilde{P}+q P, \quad \widetilde{P}=\widehat{\widetilde{P}}+e \widehat{P} .
$$

With $\Psi^{t}=(P, \widehat{P})$ we have $\widetilde{\Psi}=L \Psi$ and $\widehat{\Psi}=k^{-1} M \Psi$, where

$$
L(e, q)=\left(\begin{array}{cc}
-q & k \\
-q & k-e
\end{array}\right), \quad M(e, q, \widehat{q})=\left(\begin{array}{cc}
0 & k \\
-q & k-e+\widehat{q}
\end{array}\right) .
$$

This (small) Lax-pair differs from the (big) Lax-pair obtained in [25, equation (9)]. A big Lax-pair incorporates a particular choice of initial values and we would like to consider general periodic initial value problems. 


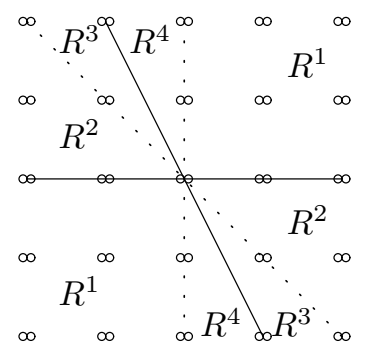

Figure 8: Distinct regions for the QD-stencil
In [38], it has been shown that for all $s=$ $\left(s_{1}, s_{2}\right) \in \mathbb{Z} \times \mathbb{Z}$ such that $s_{2}\left(s_{2}-2 s_{1}\right) \neq 0$, there exists a well-posed s-periodic initial value problem, with dimension

$$
2 \max \left(\left|s_{1}+s_{2}\right|,\left|s_{1}\right|\right)
$$

This function tells us there are two different regions, where the dimension is given by a different linear function of the periods (up to a sign). However, to pose the initial value problems one has to distinguish four different regions, as depicted in Figure 8.

We note that for all $\mathbf{s}$ initial values can be given on (part of) a standard staircase. In the Figures that follow this will be indicated by a dotted line. We will present four examples of families of periodic reductions, where the dimension depends on an arbitrary variable $n \in \mathbb{N}$, one family in each of the different regions.

\section{$(0, n)$-reduction}

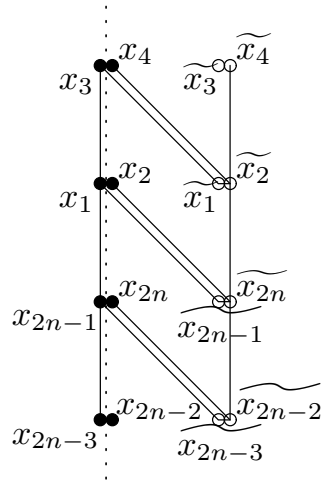

Figure 9: Vertical periodicity.
We take initial values $(e, q)_{0, i}=\left(x_{2 i-1}, x_{2 i}\right)$, with $x_{k}=x_{m}$ if $k \equiv m \bmod 2 n$, see Figure 9 .

Updating these values to the right gives a $2 n$ dimensional volume-preserving mapping, with $i=1,2, \ldots, 2 n$,

$$
\begin{aligned}
x_{2 i-1} & \mapsto x_{2 i+1}+\frac{x_{2 i+3} x_{2 i+4}}{x_{2 i+1}}-\frac{x_{2 i+1} x_{2 i+2}}{x_{2 i-1}}, \\
x_{2 i} & \mapsto \frac{x_{2 i+1} x_{2 i+2}}{x_{2 i-1}} .
\end{aligned}
$$

Here we first used equation (11b) to find the images $\widetilde{x_{2 i}}$, and then equation $(11 a)$ to find $\widetilde{x_{2 i-1}}$. The monodromy matrix is

$$
\begin{aligned}
\mathcal{L}= & M\left(x_{2 n-1}, x_{2 n}, x_{2}\right) M\left(x_{2 n-3}, x_{2 n-2}, x_{2 n}\right) \cdots \\
& \cdots M\left(x_{3}, x_{4}, x_{6}\right) M\left(x_{1}, x_{2}, x_{4}\right)
\end{aligned}
$$

We have verified up to $n=9$ that the coefficients in the $k$-expansions of its trace and determinant yield $n+1$ functionally independent integrals. The lowest nontrivial mapping, taking $n=2$, is

$$
\left(x_{1}, x_{2}, x_{3}, x_{4}\right) \mapsto\left(x_{3}+\frac{x_{1} x_{2}}{x_{3}}-\frac{x_{3} x_{4}}{x_{1}}, \frac{x_{3} x_{4}}{x_{1}}, x_{1}+\frac{x_{3} x_{4}}{x_{1}}-\frac{x_{1} x_{2}}{x_{3}}, \frac{x_{1} x_{2}}{x_{3}}\right),
$$

which admits the three functionally independent integrals $x_{1}+x_{3}, x_{2} x_{4}, x_{1} x_{2}+$ $x_{3} x_{4}-x_{1} x_{3}$. 


\section{$(3 n,-2 n)$-reduction}

We pose initial values as in Figure 10 and update then using a horse-jump $(l, m) \mapsto(l+2, m-1)$. Note that one should first update $x_{6 i+2}$ before $x_{6 i+4}$.

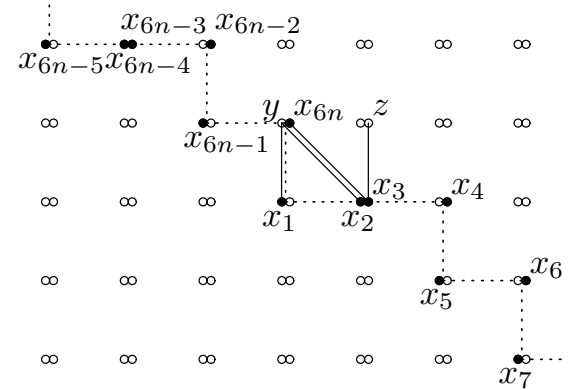

Figure 10: Initial values on part of a standard $(3 n,-2 n)$-staircase, and the image points $y, z$ of $x_{6 n-4}, x_{6 n-2}$.

Thus we get a $6 n$-dimensional mapping, with $i=0,1, \ldots, n-1$,

$$
\begin{aligned}
x_{6 i+1} & \mapsto x_{6 i+5}, \\
x_{6 i+2} & \mapsto \frac{x_{6 i+7} x_{6 i+9}}{x_{6 i+6}}, \\
x_{6 i+3} & \mapsto x_{6 i+6}, \\
x_{6 i+4} & \mapsto x_{6 i+8}+x_{6 i+9}\left(1-\frac{x_{6 i+7}}{x_{6 i+6}}\right), \\
x_{6 i+5} & \mapsto x_{6 i+8}, \\
x_{6 i+6} & \mapsto x_{6 i+10},
\end{aligned}
$$

where the subscript on $x$ is taken modulo $6 n$.

This mapping is measure-preserving with density $\left(\prod_{i=1}^{n} x_{6 i-3}\right)^{-1}$. We obtain integrals by expanding the trace and determinant of the monodromy matrix

$$
\begin{array}{r}
\prod_{i=0}^{n-1} M^{-1}\left(x_{6 i+7}, x_{6 i+5}+x_{6 i+6}-x_{6 i+7}, x_{6 i+6}\right) L\left(x_{6 i+5}, x_{6 i+2}+x_{6 i+4}-x_{6 i+5}\right) \\
\cdot M^{-1}\left(x_{6 i+5}, x_{6 i+2}+x_{6 i+4}-x_{6 i+5}, x_{6 i+4}\right) L\left(x_{6 i+2}, x_{6 i+3}\right) \\
\cdot L\left(x_{6 i+1}, x_{6 i-1}+x_{6 i}-x_{6 i+1}\right)
\end{array}
$$

in powers of the spectral parameter $k$. We verified that, up to $n=3,3 n+1$ of them are functionally independent. For $n=1$ the mapping reads

$$
\left(x_{1}, x_{2}, x_{3}, x_{4}, x_{5}, x_{6}\right) \mapsto\left(x_{5}, \frac{x_{1} x_{3}}{x_{6}}, x_{6}, x_{2}+x_{3}\left(1-\frac{x_{1}}{x_{6}}\right), x_{2}, x_{4}\right)
$$

which admits the following four functionally independent integrals $x_{6}+x_{4}+x_{2}+x_{3}, x_{3}\left(x_{4}-x_{5}\right)\left(x_{1}-x_{6}\right), x_{3}\left(x_{6}+x_{4}-x_{1}\right)+x_{6}\left(x_{4}+x_{2}-x_{5}\right), x_{1} x_{2} x_{3} x_{5}$. 
$(2 n,-3 n)$-reduction

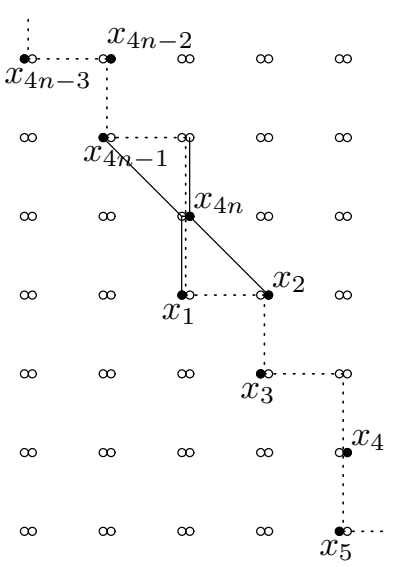

We choose initial values as in Figure 11,

$$
\begin{aligned}
e_{2 i,-3 i} & =x_{4 i+1}, \quad q_{2 i+1,-3 i}=x_{4 i+2} \\
e_{2 i+1,-3 i-1} & =x_{4 i+3}, \quad q_{2 i+2,-3 i-2}=x_{4 i+4},
\end{aligned}
$$

with $x_{k}=x_{m}$ if $k \equiv m \bmod 4 n$. They are updated by shifting $(l, m) \mapsto(l+1, m-1)$. This yields the $4 n$-dimensional mapping

$$
\begin{aligned}
x_{4 i+1} & \mapsto x_{4 i+3}, \\
x_{4 i+2} & \mapsto \frac{x_{4 i+5} x_{4 i+6}}{x_{4 i+4}}+x_{4 i+4}-x_{4 i+3}, \\
x_{4 i+3} & \mapsto \frac{x_{4 i+5} x_{4 i+6}}{x_{4 i+4}}, \\
x_{4 i+4} & \mapsto x_{4 i+6},
\end{aligned}
$$

which is measure-preserving with density $\prod_{i=1}^{n} x_{4 i}$.

The monodromy matrix is

$$
\begin{array}{r}
\prod_{i=0}^{n-1} M^{-1}\left(x_{4 i+5}, p_{i}, x_{4 i+4}\right) \\
M^{-1}\left(e_{i}, x_{4 i+4}, q_{i}\right) L\left(x_{4 i+3}, r_{i}\right) \\
\cdot M^{-1}\left(x_{4 i+3}, r_{i}, x_{4 i+2}\right) L\left(x_{4 i+1}, p_{i-1}\right),
\end{array}
$$

where $e_{i}=x_{4 i+5} x_{4 i+6} / x_{4 i+4}, q_{i}=e_{i}+x_{4 i+4}-x_{4 i+3}, r_{i}=x_{4 i+1}+x_{4 i+2}-x_{4 i+3}$, $p_{i}=z_{i}+x_{4 i+4}-x_{4 i+5}$, and $z_{i}=x_{4 i+3} r_{i} / x_{4 i+4}$. We verified up to $n=3$ that its trace and determinant yield $2 n+1$ functionally independent integrals. For $n=1$ the measure preserving 4 -dimensional mapping reads

$$
\left(x_{1}, x_{2}, x_{3}, x_{4}\right) \mapsto\left(x_{3}, \frac{x_{1} x_{2}}{x_{4}}+x_{4}-x_{3}, \frac{x_{1} x_{2}}{x_{4}}, x_{2}\right),
$$

which admits the following three functionally independent integrals,

$$
x_{2}+x_{4}-x_{3}, \frac{x_{1} x_{3}}{x_{4}}, \frac{\left(x_{1}-x_{4}\right)\left(x_{2}-x_{3}\right)\left(x_{3}-x_{4}\right)}{x_{4}} .
$$


$(1,-1-n)$-reduction

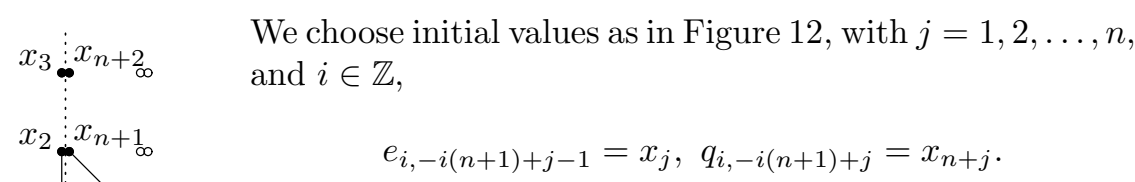

Updating by the up-shift yields a $2 n$-dimensional mapping

$$
\begin{aligned}
x_{i} & \mapsto x_{i+1}, \quad i \in\{1,2, \ldots, 2 n-1\}, i \neq n \\
x_{n} & \mapsto x_{1}+\frac{x_{2} x_{n+1}}{x_{1}}-x_{2 n}, \\
x_{2 n} & \mapsto \frac{x_{2} x_{n+1}}{x_{1}}
\end{aligned}
$$

Figure 12

which is measure-preserving with density $x_{1}$.

The monodromy matrix is

$$
\begin{aligned}
& M^{-1}\left(x_{1},\left(x_{n}+x_{2 n-1}-x_{2 n}\right) \frac{x_{2 n}}{x_{1}}, x_{n+1}\right)\left(\prod_{i=2}^{n} M^{-1}\left(x_{i}, x_{n+i-1}, x_{n+i}\right)\right) \\
& \cdot M^{-1}\left(x_{1}+\frac{x_{2} x_{n+1}}{x_{1}}-x_{2 n}, x_{2 n}, \frac{x_{2} x_{n+1}}{x_{1}}\right) L\left(x_{1},\left(x_{n}+x_{2 n-1}-x_{2 n}\right) \frac{x_{2 n}}{x_{1}}\right),
\end{aligned}
$$

whose trace and determinant yield $n+1$ functionally independent integrals, which we verified up to $n=8$. For $n=2$ we find mapping (11) again, under the change of variables

$$
\left(x_{1}, x_{2}, x_{3}, x_{4}\right) \mapsto\left(x_{4}, x_{2}, x_{1}, x_{3}\right) .
$$

\subsection{Correspondences with a sufficient number of integrals}

There are certain lines in the $\left(s_{1}, s_{2}\right)$-plane where a periodic reduction yields a correspondence instead of a mapping, see [38]. Here we impose the solution to be periodic, which is not implied by the periodicity of the initial conditions. For the QD-system, we find correspondences on lines given by $s_{2}\left(s_{2}+2 s_{1}\right)=0$, cf. $[38]$.

\subsubsection{The QD-algorithm, $(n, 0)$-reduction}

We consider horizontal staircases for the QD-system. Solving the non-local (or, implicit) scheme we find rational expressions for two-valued correspondences. 


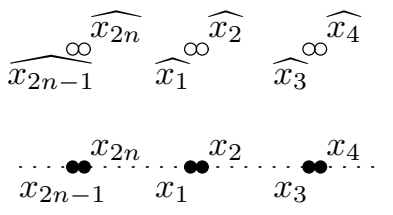

Figure 13: Initial values and their images under the up-shift.
As initial conditions we take

$$
e_{m, 0}=x_{2 m-1}, \quad q_{m, 0}=x_{2 m},
$$

where the index on $x$ is taken modulo $2 n$, see Figure 13. They are updated by the up-shift. We assume the image is periodic with the same period as the initial values, i.e. we also take the index on $\widehat{x}$ modulo $2 n$.

We have the following $2 n$ equations for the $2 n$ unknowns $\widehat{x_{i}}$ (the reader might like to draw a few of them into Figure 13)

$$
\widehat{x_{2 i-1}} \widehat{x_{2 i}}=x_{2 i-1} x_{2 i+2}, \quad \widehat{x_{2 i-1}}+\widehat{x_{2 i+2}}=x_{2 i+1}+x_{2 i+2} \text {. }
$$

We first solve for the odd variables, thereby obtaining a set of $n$ equations for the even variables

$$
\widehat{x_{2 i-1}}=\frac{x_{2 i-1} x_{2 i+2}}{\widehat{x_{2 i}}}=x_{2 i+1}+x_{2 i+2}-\widehat{x_{2 i+2}} \text {. }
$$

We write $\widehat{x_{2 i+2}}=m_{2 i+2}\left(\widehat{x_{2 i}}\right)$, where

$$
m_{k}(z)=x_{k-1}+x_{k}-\frac{x_{k-3} x_{k}}{z}
$$

Now $\widehat{x_{2 k}}$ must be one of the two fixed points of the Möbius transformation

$$
\begin{aligned}
M_{k} & =m_{2 k} m_{2(k-1)} m_{2(k-2)} \cdots m_{2(k-n+1)} \\
& =m_{2 k} \cdots m_{2(k+3)} m_{2(k+2)} m_{2(k+1)} .
\end{aligned}
$$

The first fixed point of $M_{k}$ is given by $x_{2 k-1}$, as $m_{i}\left(x_{i-3}\right)=x_{i-1}$. This gives us one way of updating our initial values, i.e. the linear map $\sigma_{2 n}$ :

$$
\begin{aligned}
x_{2 i-1} & \mapsto x_{2 i+2}, \\
x_{2 i} & \mapsto x_{2 i-1},
\end{aligned}
$$

taking $i=1,2, \ldots, n$, assuming the index on $x$ to be periodic modulo $2 n$.

The other fixed point of $M_{2 k}$ is $z_{k}:=x_{2(k+1)} Q_{2 k}^{n} / Q_{2 k-2}^{n}$, where

$$
Q_{k}^{n}(x)=\sum_{i=1}^{n-1}\left(\prod_{j=1}^{i-1} x_{2 j+2+k}\right)\left(\prod_{j=i}^{n-1} x_{2 j+1+k}\right) \text {. }
$$

This follows from

\section{Lemma 2}

$$
m_{2 k}\left(z_{k-1}\right)=z_{k}
$$


Proof: In terms of $Q$ the statement is

$$
x_{2 k-3} Q_{2 k-4}^{n}+x_{2 k+2} Q_{2 k}^{n}=\left(x_{2 k-1}+x_{2 k}\right) Q_{2 k-2}^{n}
$$

From the definition (14) it follows that

$$
x_{2 n-1} Q_{0}^{n-1}+\prod_{j=2}^{n} x_{2 j}=Q_{0}^{n}=x_{4} Q_{2}^{n-1}+\prod_{j=2}^{n} x_{2 j-1} .
$$

Therefore we have

$$
\begin{aligned}
& x_{2 n-1} Q_{-2}^{n}+x_{4} Q_{2} \\
& =x_{2 n-1}\left(x_{2} Q_{0}^{n-1}+\prod_{j=1}^{n-1} x_{2 j-1}\right)+x_{4}\left(x_{1} Q_{2}^{n-1}+\prod_{j=3}^{n+1} x_{2 j}\right) \\
& =x_{2}\left(x_{2 n-1} Q_{0}^{n-1}+\prod_{j=2}^{n} x_{2 j}\right)+x_{1}\left(x_{4} Q_{2}^{n-1}+\prod_{j=2}^{n} x_{2 j-1}\right) \\
& =\left(x_{1}+x_{2}\right) Q_{0}^{n},
\end{aligned}
$$

which is equation (15) with $k=1$. This implies that equation (15) holds for all $k$, as we may shift $x_{i} \mapsto x_{i+2(k-1)}$.

The mapping that corresponds to the fixed point $z_{k}$ will be denoted $\tau_{2 n}$ :

$$
\begin{gathered}
x_{2 i-1} \mapsto x_{2 i-1} \frac{Q_{2 i-2}^{n}}{Q_{2 i}^{n}}, \\
x_{2 i} \mapsto x_{2 i+2} \frac{Q_{2 i}^{n}}{Q_{2 i-2}^{n}} .
\end{gathered}
$$

Since a Möbius transformation has at most two fixed points we obtained a twovalued correspondence $(\sigma, \tau)$. Integrals for this correspondence are given by the coefficients of the $k$-expansions of the trace and determinant of the monodromy matrix

$$
L\left(x_{2 n}, x_{2 n-1}\right) \cdots L\left(x_{4}, x_{3}\right) L\left(x_{2}, x_{1}\right) .
$$

For all $n<10$ we found $n+1$ functionally independent integrals. The lowest non-trivial case is $n=2$. Explicitly, both mappings $\sigma_{4}$ :

$$
\left(x_{1}, x_{2}, x_{3}, x_{4}\right) \mapsto\left(x_{4}, x_{1}, x_{2}, x_{3}\right)
$$

and $\tau_{4}$ :

$$
\left(x_{1}, x_{2}, x_{3}, x_{4}\right) \mapsto\left(x_{1} \frac{x_{3}+x_{4}}{x_{1}+x_{2}}, x_{4} \frac{x_{1}+x_{2}}{x_{3}+x_{4}}, x_{3} \frac{x_{1}+x_{2}}{x_{3}+x_{4}}, x_{2} \frac{x_{3}+x_{4}}{x_{1}+x_{2}}\right)
$$

admit the three invariants $x_{1}+x_{2}+x_{3}+x_{4}, x_{1} x_{3}+x_{2} x_{4}$, and $x_{1} x_{2} x_{3} x_{4}$. 


\section{Reduction of order}

In all cases we have encountered so far, the staircase method provided a sufficient number of integrals, that is, at least half the dimension of the mapping. Still, the dimension of those mappings may be reduced. This can be done using a symmetry of the lattice equation. In certain cases the number of invariants remains the same, whereas in other cases it drops.

For reductions of other equations, which we encounter in this section, the number of integrals provided by the staircase method is not sufficient. However, in these cases, after reduction of order the number of integrals will suffice. We observe that the number of dimensions to be reduced varies with the period $\mathbf{s}$. This can be understood exploiting symmetries of the $\mathrm{P} \Delta \mathrm{E}$ that give rise, for certain periods, to $k$-symmetries of the mappings.

\subsection{Mappings with sufficiently many integrals, revisited}

\subsubsection{The Bruschi-Calogero-Droghei equation}

For the mappings obtained in section 3.1.1 the number of functionally independent integrals is exactly half the dimension of the mapping, sufficiently many for complete integrability. We note that the equation $E=0$, cf. (8), admits two Lie-point symmetries $u \mapsto u+\epsilon$ and $u \mapsto \lambda u$. This yields two symmetries for the mappings, which can be used to reduce the dimension of the mappings by 2 . The integrals we have given only admit the first (translation) symmetry. However, certain homogeneous combinations of them also admit the second (scaling) symmetry. Therefore, applying 2-reduction to the examples in section 3.1 .1 produces $2-$, respectively 4 -dimensional mappings with 1 , respectively 2 integrals. For instance, using reduced variables

$$
z_{1}=\frac{x_{1}-x_{2}}{x_{2}-x_{3}}, \quad z_{2}=\frac{x_{2}-x_{3}}{x_{3}-x_{4}},
$$

the 4-dimensional mapping (9) reduces to

$$
\left(z_{1}, z_{2}\right) \mapsto\left(z_{2}, \frac{1}{z_{1}}\right),
$$

which has one integral

$$
\frac{J_{2}^{2}}{J_{1}}=\frac{\left(z_{1}+1\right)^{2}\left(z_{2}+1\right)^{2}}{z_{1} z_{2}} .
$$

Note that the 4 th iterate of (18) equals the identity and hence the reduction provides an explicit solution for mapping (9). If the $(n-1)$ st iterate of the mapping (9) is denoted $\left(x_{n}, x_{n+1}, x_{n+2}, x_{n+3}\right)$, then

$$
\begin{aligned}
x_{n}= & x_{1}+\left\lfloor\frac{n+2}{4}\right\rfloor\left(x_{2}-x_{1}\right)+\left\lfloor\frac{n+1}{4}\right\rfloor\left(x_{3}-x_{2}\right)+\left\lfloor\frac{n}{4}\right\rfloor\left(x_{4}-x_{1}\right) \\
& +\left\lfloor\frac{n-1}{4}\right\rfloor \frac{\left(x_{2}-x_{1}\right)\left(x_{4}-x_{3}\right)}{x_{3}-x_{2}},
\end{aligned}
$$


where \lfloor\rfloor denotes the floor function, sending $x$ to the largest integer below $x$. This solution can be obtained similarly to the solution of the $(3,1)$-reduction of lattice pKdV given in the appendix of [39].

\subsubsection{The QD-algorithm}

As the QD-system (11) admits 1 (scaling) symmetry, all mappings can be 1reduced. The reduced mapping has one integral less, as only homogeneous combinations with scaling eigenvalue 0 are invariant under scaling. For example, the mapping (11) with new variables $y_{i}=x_{i} / x_{4}, i=1,2,3$, reduces to the 3 dimensional mapping

$$
\left(y_{1}, y_{2}, y_{3}\right) \mapsto\left(\frac{y_{3}}{y_{2}}, y_{1}+\frac{1-y_{3}}{y_{2}}, y_{1}\right),
$$

which has invariants

$$
I_{1}=\frac{y_{1} y_{3}}{y_{2}-y_{3}+1}, \quad I_{2}=\frac{\left(y_{2}-y_{3}\right)\left(y_{1}-1\right)\left(y_{3}-1\right)}{\left(y_{2}-y_{3}+1\right)^{2}} .
$$

This mapping has a lot of periodic points. The first few are given in Table 1 , where $a, b$ are free parameters. Also, one can show that the orbit of $(a, b, b)$ converges to the periodic orbit of $(1, a b, 1)$, which has length 3 .

\begin{tabular}{l|l} 
orbit length & periodic points \\
\hline & \\
1 & $(0,-1,0),(a, 1, a)$ \\
2 & $(0,1,2),(2,-1,0),(0, a, 0)$ \\
3 & $(1, a, 1),(1, a, a),(a, 1,1)$ \\
4 & $(a, b-1, b)$ \\
5 & $(a, b(b+1) /(a+b-1), b)$
\end{tabular}

Table 1: Periodic points with orbit length smaller than six.

Introducing variables $x=y_{1}, y=y_{3}$ on a level set of the first invariant $I_{1}=z$ yields the mapping

$$
\tau:(x, y) \mapsto\left(\frac{z y}{x y+z y-z}, x\right)
$$

This map can be written as a composition of two involutions, namely $\tau=i_{0} \circ i_{1}$, where

$$
i_{0}:(x, y) \mapsto(y, x), \quad i_{1}:(x, y) \mapsto\left(\frac{f_{1}-f_{2} x}{f_{2}-f_{3} x}, y\right)
$$

with $f=A v \times B v, v=\left(y^{2}, y, 1\right)^{t}$, which has invariant

$$
\frac{w \cdot A v}{w \cdot B v}=\frac{I_{2}}{z}
$$


where $w=\left(x^{2}, x, 1\right)$. Thus, it is a special case of the 18-parameter QRT-family of planar maps [31, 32], with

$$
A=\left(\begin{array}{ccc}
1 & -1 & 0 \\
-1 & 1-z & z \\
0 & z & -z
\end{array}\right), \quad B=\left(\begin{array}{ccc}
1 & 0 & 0 \\
0 & 0 & 0 \\
0 & 0 & 0
\end{array}\right) .
$$

Reduction of order also works for correspondences. In reduced coordinates $y_{i}=x_{i+1} / x_{1}, i=1,2,3$, the four dimensional mappings $\sigma_{4}, \tau_{4}$ reduce to

$$
\sigma:\left(y_{1}, y_{2}, y_{3}\right) \mapsto\left(\frac{1}{y_{3}}, \frac{y_{1}}{y_{3}}, \frac{y_{2}}{y_{3}}\right)
$$

and

$$
\tau:\left(y_{1}, y_{2}, y_{3}\right) \mapsto\left(y_{3} \frac{\left(y_{1}+1\right)^{2}}{\left(y_{3}+y_{2}\right)^{2}}, y_{2} \frac{\left(y_{1}+1\right)^{2}}{\left(y_{3}+y_{2}\right)^{2}}, y_{1}\right),
$$

which admit the invariants

$$
\frac{y_{2}+y_{1} y_{3}}{\left(1+y_{1}+y_{2}+y_{3}\right)^{2}}, \quad \frac{y_{1} y_{2} y_{3}}{\left(1+y_{1}+y_{2}+y_{3}\right)^{4}} .
$$

\subsection{Mappings with insufficiently many integrals}

We will next encounter reductions whose dimension is greater than twice the number of functionally independent integrals provided by the staircase method.

\subsubsection{The potential Korteweg-de Vries equation}

The matrices

$$
\left(\begin{array}{cc}
u & -k-u \widetilde{u} \\
1 & -\widetilde{u}
\end{array}\right), \quad\left(\begin{array}{cc}
u & -\alpha-k-u \widehat{u} \\
1 & -\widehat{u}
\end{array}\right),
$$

form a Lax pair for the lattice pKdV equation

$$
(u-\widehat{\widetilde{u}})(\widetilde{u}-\widehat{u})=\alpha .
$$

\subsection{2 $(n-1,1)$-reduction}

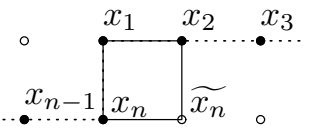

Figure 14

We consider the initial value problem

$$
u_{(n-1) k+i-1, k}=x_{i},
$$

with $i=1, \ldots, n$ and $k \in \mathbb{Z}$, see Figure 14 . We find, for all $k, u_{(n-1) k+n, k}=x_{1}+\alpha /\left(x_{n}-x_{2}\right)$.

Hence, the right-shift induces an $n$-dimensional mapping,

$$
\left(x_{1}, x_{2}, \ldots, x_{n}\right) \mapsto\left(x_{2}, x_{3}, \ldots, x_{n}, x_{1}+\frac{\alpha}{x_{n}-x_{2}}\right),
$$


which is volume-preserving when $n$ is odd and anti-volume-preserving when $n$ is even.

We have verified up to $n=17$ that the trace of the monodromy matrix provides $\lfloor(n-1) / 2\rfloor$ functionally independent integrals. So, for the odd dimensional mappings we need one reduction, but for the even dimensional mappings we need two. In fact, as we shall see, in the even case there exist three. How to explain this? As we will see below, the KdV-equation has a Lie-point symmetry that does not depend on the lattice variables. This symmetry gives rise to one reduction for both the odd and the even dimensional mappings. Also, there are two symmetries that do depend on the lattice variables. These yield two 2 -symmetries of the mapping if and only if its dimension is even, giving us two more reductions.

Equation (22) has the following symmetry $u \mapsto u+\epsilon$. This yields a symmetry for the mapping (23), whose infinitesimal generator is

$$
\sum_{i=1}^{n} \frac{\partial}{\partial x_{i}}
$$

Let $y_{i}=x_{i}-x_{i+1}$ for $i=1,2, \ldots, n-1$. The functions $y_{i}$ are annihilated by the vector field (24), they form a set of $n-1$ functionally independent invariants of the symmetry generated by this vector field. Taking the $y$ as new variables the mapping (23) reduces to

$$
\left(y_{1}, y_{2}, \ldots, y_{n-1}\right) \mapsto\left(y_{2}, y_{3}, \ldots, y_{n-1},-\sum_{i=1}^{n-1} y_{i}+\frac{\alpha}{\sum_{i=2}^{n-1} y_{i}}\right) .
$$

In addition, equation (1) also has the following two symmetries

$$
u_{l, m} \mapsto u_{l, m}-(-1)^{l+m} \epsilon, \quad u_{l, m} \mapsto u_{l, m} \epsilon^{(-1)^{l+m}} .
$$

Suppose now that $n$ is even. Then the above symmetries of the $\mathrm{P} \Delta \mathrm{E}(22)$ give rise to 2-symmetries of the mapping (25), with generators

$$
\sum_{i=1}^{n}(-1)^{i} \frac{\partial}{\partial x_{i}}, \quad \sum_{i=1}^{n}(-1)^{i} x_{i} \frac{\partial}{\partial x_{i}} .
$$

It can be verified that of $n-3$ functionally independent joint invariants of the above three vector fields $(24,26)$ are

$$
q_{i}=\left(x_{i}-x_{i+2}\right)\left(x_{i+1}-x_{i+3}\right), \quad i=1, \ldots, n-3 .
$$

We will take the $q_{i}$ as reduced variables and perform the reduction. Let us define, with $k, m \in \mathbb{N}$,

$$
F_{k}^{m}:=\frac{x_{1+k}-x_{2 m+3+k}}{x_{2 m+1+k}-x_{2 m+3+k}} .
$$


The $F_{k}^{m}$ satisfy the recurrence relation

$$
\left(F_{k}^{m}-1\right) q_{2 m+k}=q_{2 m+k-1} F_{k}^{m-1},
$$

with initial condition $F_{k}^{0}=1$. Therefore the $F_{k}^{m}$ can be expressed in the $q_{i}$, with $i \leq n-3$, when $m<n / 2-1$ and $k<n / 2-2 m+3$. We have the following equalities

$$
\begin{aligned}
x_{i} & =x_{n-1}+\frac{q_{n-3}}{x_{n-2}-x_{n}} F_{i-1}^{(n-3-i) / 2}, & & i \text { odd }, \\
x_{i} & =x_{n-2}+\frac{q_{n-4}}{q_{n-3}}\left(x_{n-2}-x_{n}\right) F_{i-1}^{(n-4-i) / 2}, & & i \text { even, }
\end{aligned}
$$

which is an inverse reduction. In terms of reduced variables the mapping is

$$
\begin{aligned}
q_{i} & \mapsto q_{i+1}, \quad i \in\{1,2, \ldots, n-4\}, \\
q_{n-3} & \mapsto-q_{n-3} F_{0}^{(n-4) / 2}+\alpha / F_{1}^{(n-4) / 2} .
\end{aligned}
$$

An explicit expression for $F_{k}^{m}$ in terms of $q_{i}$ is ${ }^{2}$

$$
F_{k}^{m}=\sum_{i=1}^{m+1} \prod_{j=2 i}^{2 m+1} q_{j-1+k}^{(-1)^{j}},
$$

since this expression solves the recurrence $(27)$ with $F_{k}^{0}=1$. The mapping (28) is anti-measure preserving with density $\prod_{i=1}^{(n-4) / 2} q_{2 i}$.

At $n=4$ the reduced mapping is $q_{1} \mapsto \alpha-q_{1}$, which admits one integral, $q_{1}\left(\alpha-q_{1}\right)$. The second iterate of this mapping equals the identity. Note that this enables one to explicitly solve equation (23) with $n=4$, cf. [39]. What happened? Well, the joint invariant $q_{1}$ turns out to be a 2-integral of the mapping. Let us define another set of functions

$$
H_{k}^{m}:=-x_{1+k} x_{2 m+2+k}-\sum_{i=1}^{2 m+1}(-1)^{i} x_{i+k} x_{i+1+k} .
$$

They can be expressed in terms of the $q_{i}$, with $i \leq n-3$, if $m<n / 2$ and $k<n / 2+4-2 m$, using the recurrence

$$
H_{k}^{m}=H_{k}^{m-1}+q_{2 m-1+k} F_{k}^{m-1},
$$

with initial condition $H_{k}^{0}=0$. For all $n$ the $n$-dimensional mapping $\delta_{n}$, see (23), admits the 2-integral $H_{0}^{(n-2) / 2}$. If the image of $x$ under (23) is denoted $\widetilde{x}$, then

$$
\widetilde{H_{0}^{(n-2) / 2}}=\alpha-H_{0}^{(n-2) / 2} \text {. }
$$

\footnotetext{
${ }^{2}$ The function $F_{k}^{m}$ is closely related to the function $Q$ given by equation (14), we have $F_{k}^{m}-1=Q_{k+1}^{m-2}(q)\left(\prod_{j=1}^{k} q_{2 j+m}\right)^{-1}$.
} 
An explicit expression for $H_{k}^{m}$ in terms of the $q_{i}$ is

$$
H_{k}^{m}=\sum_{i=1}^{m} \sum_{j=1}^{m+1-i} \prod_{l=0}^{2 i-2} q_{2 j+l-1+k}^{(-1)^{l}}
$$

since this solves the initial value problem (30). At $n=6$ the 3 -reduced mapping is

$$
\left(q_{1}, q_{2}, q_{3}\right) \mapsto\left(q_{2}, q_{3},-\frac{q_{3}\left(q_{1}+q_{2}\right)}{q_{2}}+\alpha \frac{q_{3}}{q_{2}+q_{3}}\right) .
$$

The staircase method provides two integrals

$$
q_{1} q_{3}\left(q_{2}+q_{3}-\alpha+\frac{q_{1}\left(q_{2}+q_{3}\right)}{q_{2}}\right),\left(q_{1}+q_{3}+\frac{q_{1} q_{3}}{q_{2}}\right)\left(q_{1}+q_{3}-\alpha+\frac{q_{1} q_{3}}{q_{2}}\right)
$$

of which the latter can be expressed in terms of the 2-integral $H_{0}^{2}=q_{1}+$ $q_{3}+q_{1} q_{3} / q_{2}$. We can take the 2 -integral as a variable. In terms of $q_{1}, q_{2}$ and $p=H_{0}^{(n-2) / 2}$ mapping (31) becomes

$$
\left(q_{1}, q_{2}, p\right) \mapsto\left(q_{2}, q_{2}\left(p-q_{1}\right) /\left(q_{1}+q_{2}\right), \alpha-p\right)
$$

which has integrals

$$
\frac{q_{1} q_{2}}{q_{1}+q_{2}}\left(p-q_{1}\right)\left(p+q_{2}-\alpha\right), p(\alpha-p) .
$$

In general, with $n>2$ even, the 3-reduced mapping can be written as

$$
\left(q_{1}, q_{2}, \ldots, q_{n-4}, p\right) \mapsto\left(q_{2}, q_{3}, \ldots, q_{n-4}, \frac{p-H_{0}^{(n-4) / 2}}{F_{0}^{(n-4) / 2}}, \alpha-p\right),
$$

where we used (30) to solve $p=H_{0}^{(n-2) / 2}$ for $q_{n-3}$.

We note that all $\lfloor(n-1) / 2\rfloor$ functionally independent integrals we have calculated for $n \leq 17$ dimensional mappings survive these reductions. Taking $n=2 m+1$ odd, the reduced mapping is $2 m$ dimensional and has $m$ integrals. With $n=2 m+2$, the reduced mapping is $2 m-1$ dimensional and has $m$ integrals. 


\subsection{The Boussinesq system}

$$
\begin{array}{rlrl}
u & u & \text { The Boussinesq system }[22,35] \\
& \widetilde{w} & =u \widetilde{u}-v \\
\widehat{w} & =u \widehat{u}-v \\
w & w & =u \widehat{\widetilde{u}}-\widehat{\widetilde{v}}+\frac{\gamma}{\widetilde{u}-\widehat{u}} .
\end{array}
$$

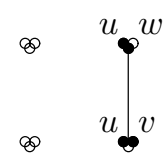

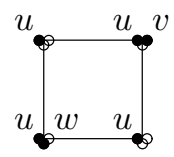

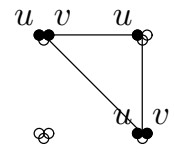

Figure 15 $(34 \mathrm{a}, 34 \mathrm{~b})$,

$$
\widehat{u} \widehat{\widetilde{u}}-\widehat{v}=\widetilde{u} \widehat{\widetilde{u}}-\widetilde{v} .
$$
$v$ and $w$ using the identity

$$
(\widetilde{\widehat{v}}+w)^{\widetilde{v}}-(\widetilde{\widehat{v}}+w) \tilde{}=(\widehat{v}-\widetilde{v})^{\widetilde{T}}+(\widehat{w}-\widetilde{w})
$$

is defined on the square as depicted in Figure 15(a,b,c). In Figure 15(d) we have depicted the consequence of equations

From the Boussinesq system one can eliminate the variables

to get a 9 -point scalar equation on a $2 \times 2$ square, called the Boussinesq equation, cf. [22, equation 1.3].

We denote $\mathbf{u}=(u, v, w)$. A Lax-pair for the system (34) is given by, cf. [35],

$$
\begin{aligned}
L_{\mathbf{u}} & =L(u, w, \widetilde{u}, \widetilde{v})=\left(\begin{array}{ccc}
-\widetilde{u} & 1 & 0 \\
-\widetilde{v} & 0 & 1 \\
\widetilde{u} w-\widetilde{v} u-k & -w & u
\end{array}\right), \\
M_{\mathbf{u}} & =M(u, w, \widehat{u}, \widehat{v})=\left(\begin{array}{ccc}
-\widehat{u} & 1 & 0 \\
-\widehat{v} & 0 & 1 \\
\widehat{u} w-\widehat{v} u+\gamma-k & -w & u
\end{array}\right) .
\end{aligned}
$$

Invariants for traveling wave reductions of the system can be obtained by expanding traces of powers of the monodromy matrix. Since $\mathcal{L}$ is a $3 \times 3$ matrix a full set of functionally independent integrals can be obtained from $k$-expansions of the coefficients in

$$
\begin{aligned}
\operatorname{Det}(\lambda I-\mathcal{L})= & \lambda^{3}-\lambda^{2} \operatorname{Tr}(\mathcal{L})+\lambda \frac{\operatorname{Tr}(\mathcal{L})^{2}-\operatorname{Tr}\left(\mathcal{L}^{2}\right)}{2} \\
& -\frac{\operatorname{Tr}(\mathcal{L})^{3}-3 \operatorname{Tr}(\mathcal{L}) \operatorname{Tr}\left(\mathcal{L}^{2}\right)+2 \operatorname{Tr}\left(\mathcal{L}^{3}\right)}{6} .
\end{aligned}
$$

cf. section 2, in particular equation (7). However, due to the fact that both Lax-matrices have a constant determinant, it suffices to consider $\operatorname{Tr}(\mathcal{L})$ and $\operatorname{Tr}^{2}(\mathcal{L})-\operatorname{Tr}\left(\mathcal{L}^{2}\right)$.

The following proposition tells us how to pose initial value problems for the Boussinesq system. The proof uses a different technique than the one used in [38], which is possible due to the fact that for the Boussinesq system initial values can be given on staircases. However, the staircases are not necessarily 
standard staircases, which they would be in the framework of [38] (at least for equations defined on the square, such as the Boussinesq system).

We call a staircase ascending, if it goes from the lower left to the upper right, that is, if it is a sequence of neighboring lattice sites with $l$ and $m$ nondecreasing. And we call a staircase descending if it goes from the upper left to the lower right, that is, if it is a sequence of neighboring lattice sites with $l$ and $-m$ nondecreasing.

Proposition 3 The following initial values problems for the Boussinesq system are well-posed.

- At every point on an ascending staircase take the components $u, v$ of the vector $\mathbf{u}$ as initial values.

- On a descending staircase take $u, v, w$ at the lower left corners, $v$ at the upper right corners, and $u, v$ at the other points as initial values.

Proof: The proof consist of two parts. Firstly, we show that the values at all points of the staircase can be obtained from the initial values. Secondly we show that any of the four vectors $\mathbf{u}, \widetilde{\mathbf{u}}, \widehat{\mathbf{u}}, \widehat{\widetilde{\mathbf{u}}}$, can be determined from the other three.

i) For ascending staircases the first part is easy. Going along the staircase from the lower-left to the upper-right at each horizontal step the component $w$ is obtained using equation (34a), whereas at the vertical steps equation (34b) can be used. For a descending staircase we can do a similar thing, except at the upper-right corners. Equation (34d) can be solved for $u_{l+1, m+1}$ and used to get the $u$-components at the upper-right corners. Once $u$ has been calculated $w$ can be calculated in two ways, using either $(34 a)$ or $(34 \mathrm{~b})$, leading to the same result.

ii) The values of $\widehat{\mathbf{u}}$ can be obtained as follows. First calculate $\widehat{u}$ from (34c). Then $\widehat{w}$ can be obtained from $(34 b)$ and $\widehat{v}$ from the up-shifted consequence of $(34 a)$. We can obtain $\widetilde{\mathbf{u}}$ in a similar way. This follows from the fact that interchanging the left-shift with the up-shift and $\gamma \mapsto-\gamma$ is a (discrete) symmetry of the system. Finally, to obtain $\widehat{\widetilde{\mathbf{u}}}$ one uses the consequence $(34 d)$ to calculate $\widehat{\widetilde{u}}$, after which $\widehat{\widetilde{w}}$ is found using a shifted version of either $(34 a)$ or $(34 b)$, and $\widehat{\widetilde{v}}$ is calculated using (34c). Finally, due to the discrete symmetry which interchanges the up-shift with the down-shift and the left-shift with the right-shift, together with $u \leftrightarrow w$ and $\gamma \mapsto-\gamma$, it follows that $\mathbf{u}$ can be obtained from given values at the other sites.

It follows that, with $s=\left(s_{1}, s_{2}\right) \in \mathbb{Z} \times \mathbb{Z}$ such that $s_{1} s_{2} \neq 0$, the dimension of an s-periodic reduction is $2\left(\left|s_{1}\right|+\left|s_{2}\right|\right)$. 


\section{(n-1,1)-reduction}

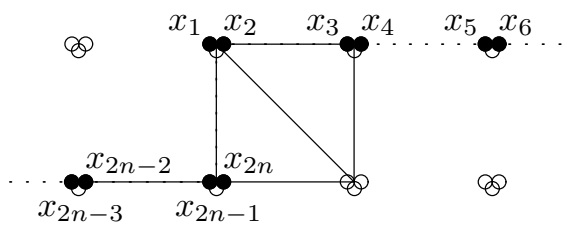

Figure 16: $2 n$ initial values
We take $s_{1}=n-1$ positive (but $n \neq 3), s_{2}=1$, and we consider the following initial value problem, with $i=0,1, \ldots, n-1$ and $l \in \mathbb{Z}$.

$$
\begin{aligned}
& u_{(n-1) l+i, l}=x_{2 i+1}, \\
& v_{(n-1) l+i, l}=x_{2 i+2},
\end{aligned}
$$

where the index on $x$ is taken modulo $2 n$, see Figure 16.

Using the equations $(34 a),(34 c)$, and $(34 d)$, in that order, we calculate, $w_{(n-1) l, l-1}=$ $x_{2 n-3} x_{2 n-1}-x_{2 n-2}, u_{(n-1) l+1, l-1}=x_{1}+\gamma P$, and $v_{(n-1) l+1, l k-1}=x_{2}+x_{3} \gamma P$, where

$$
P=\frac{1}{x_{2 n-1}\left(x_{2 n-3}-x_{3}\right)-x_{2 n-2}+x_{4}} .
$$

The right-shift induces the $2 n$-dimensional mapping $\phi_{2 n}$ :,

$$
\begin{aligned}
x_{i} & \mapsto x_{i+2}, \quad i \in\{1,2, \ldots, 2 n-2\}, \\
x_{2 n-1} & \mapsto x_{1}+\gamma P, \\
x_{2 n} & \mapsto x_{2}+\gamma x_{3} P .
\end{aligned}
$$

The monodromy matrix is

$$
\mathcal{L}=M\left(x_{2 n-1}, w_{n}, x_{1}, x_{2}\right) L\left(x_{2 n-3}, w_{n-1}, x_{2 n-1}, x_{2 n}\right) \cdots L\left(x_{1}, w_{1}, x_{3}, x_{4}\right),
$$

where $w_{1}=x_{1} x_{2 n-1}-x_{2 n}$ and $w_{i+1}=x_{2 i-1} x_{2 i+1}-x_{2 i}, i=1,2, \ldots, n-1$.

\begin{tabular}{l|l|l|l|l|l|l|l|l}
$n$ & 2 & 3 & 4 & 5 & 6 & 7 & 8 & 9 \\
\hline$\#$ & 1 & 0 & 3 & 4 & 3 & 6 & 7 & 6
\end{tabular}

The number of functionally independent integrals we have obtained for reductions with period $\mathbf{s}=(n-1,1)$

Table 2: Number of functionally inis given in table 2. From this table dependent integrals of $\phi_{2 n}$.

it seems we need to $d$-reduce mapping $\phi_{2 n}$ by $d=2$ dimensions, or, if 3 divides $n$, by $d=6$ dimensions.

The mapping $\phi_{2 n}$ has two symmetries, generated by

$$
v_{1}=\sum_{i=1}^{n} \frac{\partial}{\partial x_{2 i}}, \quad v_{2}=\sum_{i=1}^{n} \frac{\partial}{\partial x_{2 i-1}}+x_{2 i-1} \frac{\partial}{\partial x_{2 i}} .
$$

The easiest way to check that these vector fields are generators of symmetries indeed is using the Jacobian, we have $J v=\phi_{2 n}(v)$ when $v=(0,1,0,1, \ldots, 0,1)$ or $v=\left(1, x_{1}, 1, x_{3}, \ldots, 1, x_{2 n-1}\right)$, where the Jacobian matrix of $\phi_{2 n}$ is given by

$$
J=\left(\begin{array}{cc}
0 & I_{2 n-2} \\
I_{2} & \gamma P^{2} H
\end{array}\right)
$$


where $I_{k}$ is the $k \times k$ identity matrix and $H$ is the $2 \times(2 n-2)$ matrix

$$
\left(\begin{array}{ccccccccc}
x_{2 n-1} & -1 & 0 & \cdots & 0 & -x_{2 n-1} & 1 & \left(x_{3}-x_{2 n}-3\right) & 0 \\
1 / P+x_{3} x_{2 n-1} & -x_{3} & 0 & \cdots & 0 & -x_{3} x_{2 n-1} & x_{3} & x_{3}\left(x_{3}-x_{2 n}-3\right) & 0
\end{array}\right) .
$$

The two symmetries of the mapping $\phi_{2 n}$ correspond to the following symmetries of the original lattice system (34):

$$
(u, v, w) \mapsto(u, v+\epsilon, w-\epsilon), \quad(u, v, w) \mapsto(u+\epsilon, v+\epsilon u, w+\epsilon u)
$$

It can be verified that the functions

$$
y_{i}=x_{2 i-1}-x_{2 i+1}, y_{n+i-1}=x_{2 i}-x_{2 i+2}+x_{2 i-1}\left(x_{2 i+1}-x_{2 i-1}\right),
$$

with $i=1, \ldots, n-1$, are joint invariants of the these symmetries and functionally independent. In the reduced variables $y$, we get a $2(n-1)$-dimensional volume preserving mapping

$$
\begin{aligned}
y_{i} & \mapsto y_{i+1}, \quad i \in\{1,2, \ldots, 2 n-2\}, i \neq n-1, \\
y_{n-1} & \mapsto-\sum_{i=1}^{n-1} y_{i}-\gamma Q, \\
y_{2 n-2} & \mapsto-\left(\sum_{i=0}^{n-2} y_{n+i}+y_{i+1} \sum_{j=i+1}^{n-1} y_{j}\right)-\gamma Q \sum_{i=2}^{n-1} y_{i},
\end{aligned}
$$

where $Q=\sum_{i=1}^{n-3}\left(y_{n+i}+y_{i+1} \sum_{j=i+1}^{n-1} y_{j}\right)$.

When $n=2$ the reduced mapping is, in terms of $X=-y_{1}, Y=-y_{2}$

$$
(X, Y) \mapsto\left(-X+\gamma\left(Y-X^{2}\right),-Y+X^{2}\right)
$$

which carries the invariant $Y\left(X^{2}-Y\right)+\gamma X$, cf. [22, equation 5.31]. Note, in [22] the case $s_{1}=s_{2}$ was studied, in particular the involutivity of the integrals was established in any dimension. The authors defer the actual counting of independent integrals to a future study. However, they also state that the investigation of lower-dimensional examples (with $s_{1}=s_{2}$ ) indicate a sufficient number of invariants are functionally independent.

We have verified that all integrals we found, see Table 2, survive the 2reduction. Hence, in those cases, except when 3 divides $n$, the staircase method provides enough integrals for integrability. Next we will show that if 3 divides $n$, but $n \neq 3$, we can further reduce the mapping by four dimension.

The Boussinesq system (34) has some additional symmetries, which depend on the lattice variables $\left(\mathbf{u}=\mathbf{u}_{l, m}\right)$,

$$
\begin{aligned}
(u, v, w) & \mapsto\left(u, v+\epsilon \zeta^{l+m+1}, w-\epsilon \zeta^{l+m}\right) \\
(u, v, w) & \mapsto\left(u+\epsilon \zeta^{l+m}, v+\epsilon \zeta^{l+m+1} u, w+\epsilon \zeta^{l+m-1} u\right)
\end{aligned}
$$


where $\zeta$ is a primitive third root of unity, that is, $\zeta^{2}+\zeta+1=0$. The generators of the corresponding transformations acting on the initial values (16) are

$$
v_{3}=\sum_{i=1}^{n} \zeta^{i} \frac{\partial}{\partial x_{2 i}}, \quad v_{4}=\sum_{i=1}^{n}\left(\zeta^{i-1} \frac{\partial}{\partial x_{2 i-1}}+\zeta^{i} x_{2 i-1} \frac{\partial}{\partial x_{2 i}}\right)
$$

and, taking the conjugate root $\zeta^{2}$,

$$
v_{5}=\sum_{i=1}^{n} \zeta^{2 i} \frac{\partial}{\partial x_{2 i}}, \quad v_{6}=\sum_{i=1}^{n}\left(\zeta^{2 i+1} \frac{\partial}{\partial x_{2 i-1}}+\zeta^{2 i} x_{2 i-1} \frac{\partial}{\partial x_{2 i}}\right) .
$$

Now assume that 3 divides $n$. We construct real vector fields by taking the following linear combinations, with $i=1,2,3$,

$$
\begin{aligned}
w_{i} & =\left(v_{1}+\zeta^{2 i} v_{3}+\zeta^{i} v_{5}\right) / 3=\sum_{j=0}^{n / 3-1} \frac{\partial}{\partial x_{6 j+2 i}} \\
w_{3+i}=\left(v_{2}+\zeta^{2 i} v_{4}+\zeta^{i} v_{6}\right) / 3 & =\sum_{j=0}^{n / 3-1} x_{6 j+2 i-1} \frac{\partial}{\partial x_{6 j+2 i}}+\frac{\partial}{\partial x_{6 j+2 i+1}},
\end{aligned}
$$

where $x_{2 n+1}=x_{1}$. These vector fields are 3-symmetries of the mapping $\phi_{2 n}$. Let $J^{3}$ be the Jacobian matrix of $\phi_{2 n}^{3}$. Note, the vector fields $w_{i}, i \neq 1,4$ can be obtained from $w_{i}=J^{3} \phi_{2 n}\left(w_{i-1}\right)$. According to [13, proposition 1] it suffices to verify that $w_{1}$ and $w_{4}$ are 3 -symmetries. Also note that $J^{3} \phi_{2 n} v_{i}=v_{i}$ for $i \in\{1, \ldots, 6\}$.

The following polynomials form a complete set of joint invariants of the vector fields $w_{1}, w_{2}, \ldots, w_{6}$ :

$$
\begin{aligned}
z_{i}= & x_{2 i-1}-x_{2 i+5}, \quad i \in\{1, \ldots, n-3\}, \\
z_{n-3+i}= & x_{2 i+1}\left(x_{2 i-1}-x_{2 i+5}\right)-x_{2 i}+x_{2 i+6}, \quad i \in\{1,2,3\} \\
z_{n+i}= & \left(x_{2 i-1}-x_{2 i+5}\right)\left(x_{2 i+6}-x_{2 i+12}\right) \\
& -\left(x_{2 i}-x_{2 i+6}\right)\left(x_{2 i+5}-x_{2 i+11}\right), \quad i \in\{1,2, \ldots, n-6\} .
\end{aligned}
$$

Another joint invariant is given by $P$, see (35), which therefore should be expressible in terms of the $z_{i}$. However, we haven't found a general formula for $P(z)$. In terms of the $z$-variables the mapping $\phi_{12}$ reduces to

$$
\begin{aligned}
z_{i} & \mapsto z_{i+1}, \quad i \in\{1,2,4,5\} \\
z_{3} & \mapsto-z_{1}+\frac{\gamma}{z_{5}-z_{2} z_{3}}, \\
z_{6} & \mapsto-z_{4}+z_{1} z_{2}-\gamma \frac{z_{2}}{z_{5}-z_{2} z_{3}},
\end{aligned}
$$


and $\phi_{2 n}$, with 3 divides $n>4$,

$$
\begin{aligned}
z_{i} & \mapsto z_{i+1}, \quad i \in\{1,2, \ldots, 2 n-7\}, i \neq n-3, i \neq n \\
z_{n-3} & \mapsto-\sum_{i=1}^{n / 3-1} z_{3 i-2}-\gamma P, \\
z_{n} & \mapsto-\frac{z_{n+1}+z_{4}\left(z_{1} z_{2}-z_{n-2}\right)}{z_{1}}, \\
z_{2 n-6} & \mapsto A-\gamma B P,
\end{aligned}
$$

where $A=\left(x_{2 n-11}-x_{2 n-5}\right)\left(x_{2 n-4}-x_{2}\right)-\left(x_{2 n-10}-x_{2 n-4}\right)\left(x_{2 n-5}-x_{1}\right)$, and $B=x_{3}\left(x_{2 n-11}-x_{2 n-5}\right)-x_{2 n-10}+x_{2 n-4}$ are also joint invariants of the six vector fields $w_{i}$. All the integrals we found survive the above reduction. Thus, as one can see in Table 2, also when 3 divides $n$ the staircase method provides a sufficient number of integrals for the 6-reduced map to be integrable.

We found two functions which are 3-integrals, that is, integrals of $\phi_{2 n}^{3}$. These are

$$
\begin{aligned}
i_{1} & =\sum_{j=1}^{n / 3}\left(x_{6 j+1}-x_{6 j-5}\right)\left(x_{6 j-2}-x_{6 j-3} x_{6 j-1}\right)+x_{6 j-1}\left(x_{6 j+2}-x_{6 j-4}\right), \\
i_{2} & =\sum_{j=1}^{n / 3} x_{6 j-1}\left(x_{6 j-5} x_{6 j-3}-x_{6 j-4}-x_{6 j+1} x_{6 j+3}+x_{6 j+2}\right)+x_{6 j}\left(x_{6 j+3}-x_{6 j-3}\right) .
\end{aligned}
$$

We have the following action of $\phi_{2 n}$ on the 3 -integrals

$$
\left(i_{1}, i_{2}\right) \mapsto\left(i_{2}-i_{1}+\gamma,-i_{1}+\gamma\right),
$$

whose third power is the identity. Note that by applying the map to one of the 3-integrals gives us the other one but no third functionally independent 3integral can be obtained in this way. The 3 -integrals admit the vector fields $w_{i}$ as symmetries and two of the $n-3$ functionally independent integrals found by the staircase method can be written in terms of them. For $n=6$ we have

$$
i_{1}=-z_{1} z_{2} z_{3}+z_{1} z_{5}+z_{3} z_{4}, \quad i_{2}=z_{2} z_{6}+z_{3} z_{4} .
$$

and two of the three functionally independent integrals found by the staircase method are given by

$$
i_{2}^{2}+i_{1}\left(i_{1}-i_{2}-\gamma\right), i_{1}\left(\gamma-i_{2}\right)\left(\gamma-i_{1}+i_{2}\right)
$$

which are both integrals of the 2-dimensional map (39). An extra advantage of working with expansion (34) instead of traces of powers of $\mathcal{L}$ is that the third functionally independent integral factorizes nicely as

$$
z_{4} z_{5} z_{6}\left(z_{1} z_{3}+z_{6}\right)\left(z_{4} z_{5}-z_{4} z_{2} z_{3}-z_{2} z_{1} z_{5}+z_{2}^{2} z_{1} z_{3}+\alpha z_{2}\right) .
$$




\section{$(n-1,-1)$-reduction}

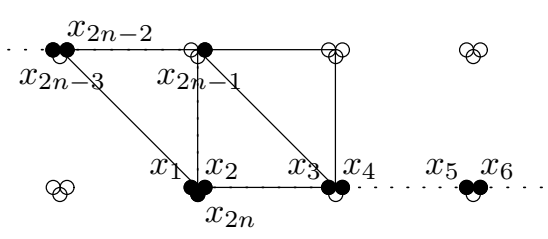

Figure 17: Periodic initial value problem for the Boussinesq system

We take $s_{1}=n-1$ positive $(n \neq 2)$, $s_{2}=-1$, and we consider the following initial value problem, with $i=$ $0,1, \ldots, n-2, k \in \mathbb{Z}$

$$
\begin{aligned}
u_{(n-1) k+i,-k} & =x_{2 i+1}, \\
v_{(n-1) k+i,-k} & =x_{2 i+2}, \\
v_{(n-1)(k+1),-k} & =x_{2 n-1}, \\
w_{(n-1) k,-k} & =x_{2 n},
\end{aligned}
$$

see Figure 17.

We right-shift the initial values $x_{2 n-3}$ and $x_{2 n}$ using equations (34d) and (34a), respectively. Then we right-shift $x_{2 n-3}$ a second time and are able to determine the image of $x_{2 n-1}$, using equation (34c). Thus, we find the $2 n$-dimensional mapping $\zeta_{2 n}$ :

$$
\begin{aligned}
x_{i} & \mapsto x_{i+2}, \quad i \in\{1,2, \ldots, 2 n-3\}, \\
x_{2 n-3} & \mapsto \frac{x_{2 n-2}-x_{2}}{x_{2 n-3}-x_{1}}, \\
x_{2 n-2} & \mapsto x_{2 n-1}, \\
x_{2 n-1} & \mapsto-x_{2 n}+\frac{\left(x_{2 n-3}-x_{1}\right)\left(x_{1}\left(x_{2 n-1}-x_{4}\right)-\gamma\right)}{x_{2 n-2}-x_{2}-x_{3}\left(x_{2 n-3}-x_{1}\right)}, \\
x_{2 n} & \mapsto-x_{2}+x_{1} x_{3},
\end{aligned}
$$

which is measure preserving with density $x_{2 n-3}-x_{1}(n \neq 2)$. The mapping $\zeta_{2 n}$ admits the symmetries

$$
\frac{\partial}{\partial x_{2 n-1}}-\frac{\partial}{\partial x_{2 i}}+\sum_{i=1}^{n-1} \frac{\partial}{\partial x_{2 i}}
$$

and

$$
x_{1} \frac{\partial}{\partial x_{2 n}}+\zeta_{2 n}\left(x_{2 n-3}\right) \frac{\partial}{\partial x_{2 n-1}}+\sum_{i=1}^{n-1}\left(\frac{\partial}{\partial x_{2 i-1}}+x_{2 i-1} \frac{\partial}{\partial x_{2 i}}\right) .
$$

Hence, it can be reduced to a $(2 n-2)$-dimensional mapping. We have verified up to $n=7$, that the number of functionally independent integrals, obtained by $k$-expansion of the coefficients in (34), with

$$
\begin{array}{r}
\mathcal{L}=M^{-1}\left(x_{1}, x_{2 n}, \zeta_{n}\left(x_{2 n-3}\right), x_{2 n-1}\right) L\left(x_{2 n-3}, w_{n-1}, \zeta_{n}\left(x_{2 n-3}\right), x_{2 n-1}\right) \\
\cdot \prod_{i=2}^{n-1} L\left(x_{2 i-3}, w_{i-1}, x_{2 i-1}, x_{2 i}\right),
\end{array}
$$

is $n-1$, except when 3 divides $n+1$, where the number is $n-3$. Also we verified that all these integrals admit the above vector fields as their symmetries. If 3 
divides $n+1$ the symmetries (38) yield 3-symmetries of the mapping $\zeta_{2 n}$. As in the previous example we take linear combinations to get

$w_{1}=\sum_{j=0}^{(n-2) / 3} \frac{\partial}{\partial x_{6 j+2}}, w_{4}=\left(\sum_{j=0}^{(n-5) / 3-1} x_{6 j+1} \frac{\partial}{\partial x_{6 j+2}}+\frac{\partial}{\partial x_{6 j+3}}\right)+x_{2 n-3} \frac{\partial}{\partial x_{2 n-2}}$,

together with $w_{i}=J^{3} \zeta_{2 n}\left(w_{i-1}\right), i \neq 1,4$. Taking $n=5$ we obtain the following 4-dimensional 6-reduced mapping, in terms of $y_{1}=x_{7}-x_{1}, y_{2}=x_{6}+x_{10}-$ $x_{5} x_{1}, y_{3}=x_{8}-x_{2}+x_{3}\left(-x_{7}+x_{1}\right), y_{4}=\left(-x_{7}+x_{1}\right)\left(-x_{9}+x_{4}-x_{5} x_{3}\right)+x_{5}\left(-x_{8}+x_{2}\right)$ :

$$
\left(y_{1}, y_{2}, y_{3}, y_{4}\right) \mapsto\left(\frac{y_{3}}{y_{1}}, y_{3}, \frac{y_{4}}{y_{1}}, y_{4}+\gamma-\frac{y_{2} y_{3}}{y_{1}}\right) .
$$

This mapping is measure preserving with density $y_{1}^{2}$. It has two 3 -integrals, $i_{1}=y_{2} y_{3} / y_{1}$ and $i_{2}=y_{4}$, which satisfy

$$
\left(i_{1}, i_{2}\right) \mapsto\left(i_{2},-i_{1}-i_{2}-\gamma\right)
$$

whose third power is the identity. The two functionally independent invariants found by the staircase method can be expressed in terms of the 3-integrals as

$$
i_{1}^{2}+i_{2}^{2}+i_{1} i_{2}+\gamma\left(i_{1}+i_{2}\right), \quad i_{1} i_{2}\left(i_{1}+i_{2}+\gamma\right) .
$$

\subsubsection{The potential Korteweg-De Vries equation, (3,0)-reduction}

Consider initial values for the pKdV equation (22): $u_{l, 0}=x_{k}$ with $k \equiv l \bmod$ $3, k \in\{1,2,3\}$, as in Figure 18 .

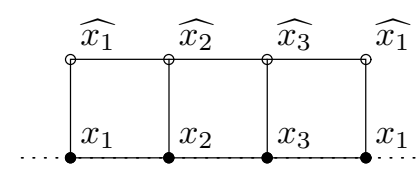

Figure 18: Initial values on a horizontal staircase with periodicity $u_{l, m}=u_{l+3, m}$.
Updating these by shifting them upwards, and imposing the image to be periodic, we have to solve the system

$$
\begin{aligned}
& \left(x_{1}-\widehat{x_{2}}\right)\left(x_{2}-\widehat{x_{1}}\right)=\alpha \\
& \left(x_{2}-\widehat{x_{3}}\right)\left(x_{3}-\widehat{x_{2}}\right)=\alpha \\
& \left(x_{3}-\widehat{x_{1}}\right)\left(x_{1}-\widehat{x_{3}}\right)=\alpha
\end{aligned}
$$

for the image points $\widehat{x_{1}}, \widehat{x_{2}}, \widehat{x_{3}}$.

In terms of reduced variables $q_{1}=x_{1}-x_{2}, q_{2}=x_{2}-x_{3}$ the volume preserving two-valued correspondence is $\varphi_{ \pm}$:

$$
\begin{aligned}
& q_{1} \mapsto q_{1} \frac{\alpha\left(\alpha+q_{2}\left(q_{1}+q_{2}\right)\right)+q_{1}^{2} q_{2}\left(q_{1}+q_{2}\right) / 2+\left(q_{1} / 2+q_{2}\right) R}{\left(\alpha+q_{1} q_{2}\right)\left(\alpha-q_{1}\left(q_{1}+q_{2}\right)\right)} \\
& q_{2} \mapsto q_{2} \frac{\alpha\left(\alpha+q_{1}\left(q_{1}+q_{2}\right)\right)+q_{1} q_{2}^{2}\left(q_{1}+q_{2}\right) / 2-\left(q_{1}+q_{2} / 2\right) R}{\left(\alpha+q_{1} q_{2}\right)\left(\alpha-q_{2}\left(q_{1}+q_{2}\right)\right)},
\end{aligned}
$$

where

$$
R= \pm \sqrt{4 \alpha^{3}+\left(q_{1} q_{2}\left(q_{1}+q_{2}\right)\right)^{2}} .
$$

This correspondence admits the integral $q_{1} q_{2}\left(q_{1}+q_{2}\right)$, which can be obtained by taking the trace of the monodromy matrix. 


\section{The staircase method on quad-graphs}

Recently, in [4], a geometric criterion was given for the well-posedness of initial value problems on quad-graphs. In this section we show that for 'regular' quad-graphs, those that permit periodic solutions, the staircase method can be applied. We use equation $H 3_{\delta=0}$, from [2],

$$
Q_{p q}(a, b, c, d):=p(a b+c d)-q(a c+b d)=0,
$$

on two different quad-graphs, cf. Figure 9d and 9e in [4].

We start with a brief introduction to the idea of a quad-graph. For a more thorough treatment and references to the literature we refer to [4]. A quad-graph is a planar graph with quadrilateral faces. Fields are assigned to the vertices and parameters to its edges. In the class considered in [4] opposite edges carry the same parameters and the (multi-linear) equation is supposed to have $D_{4^{-}}$ symmetry (so that the equation can be defined on each face independently of its position in the quad-graph). Due to the first property there are sequences of adjacent quadrilaterals on which the value of the parameter is constant. These are called characteristics. The main result in [4] states that an initial value problem $P$ is well-posed if and only if each characteristic intersects $P$ in exactly one edge.

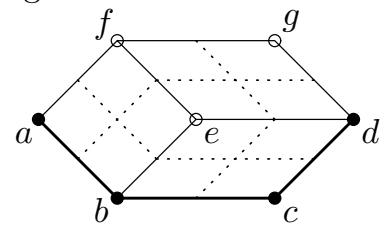

Figure 19

An example of a well-posed initial value problem is given in Figure 19: from given values $a, b, c, d$ one can calculate $e, f, g$ successively, they are uniquely determined. Indeed, the three dotted lines are characteristics and they each intersect the initial value path in exactly one edge.

In both Figures 20 and 21 a finite piece of a quad-graph is given. We assume these quad-graphs extend periodically in both the vertical and horizontal directions. For equations on such a doubly infinite quad-graph there exist a two-parameter family of periodic reductions, as for equations on the regular $\mathbb{Z}^{2}$ lattice. In fact, these quad-graphs are perturbations of the regular $\mathbb{Z}^{2}$-lattice, we call them irregular $\mathbb{Z}^{2}$-lattices.

Theorem 1 extends to the more general setting of irregular $\mathbb{Z}^{2}$-lattices. The proof is similar as in the $\mathbb{Z}^{2}$ setting: the fact the transfer matrix $\mathcal{L}_{a, b}$ does not depend on the path from $a$ to $b$ follows from the Lax-condition. 


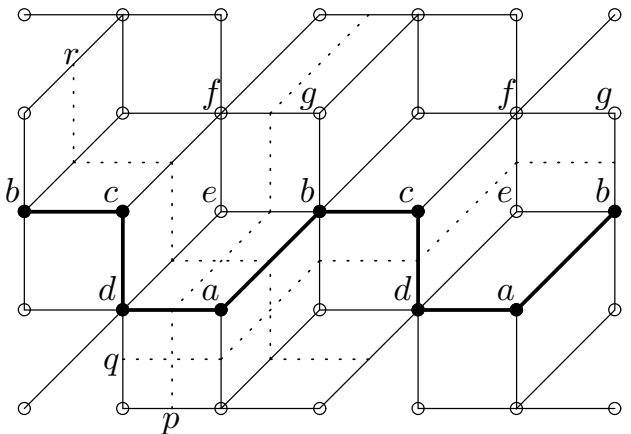

Figure 20
In Figure 20 the lattice parameters are attached to the edges as follows: $p$ to the horizontal edges, $q$ to the vertical edges, and $r$ to the diagonal edges. From initial values $a, b, c, d$ the values $e, f, g$ are determined by

$$
\begin{aligned}
& Q_{p r}(d, a, e, b)=0, \\
& Q_{q r}(c, d, f, e)=0, \\
& Q_{p q}(e, b, f, g)=0 .
\end{aligned}
$$

We update values by shifting over $(2,1)$. Thus, the mapping is

$$
(a, b, c, d) \mapsto(c, f, g, b),
$$

where

$$
f=d \frac{a r(r b-p d)+q c(r d-p b)}{a q(+r b-p d)+r c(r d-p b)}, \quad g=a \frac{r b(q a-p c)+q d(q c-a p)}{q b(q a-p c)+r d(q c-a p)} .
$$

A Lax pair $L, M$ for equation (41) is given by $L=L_{a, b}(p), M=L_{a, c}(q)$, where

$$
L_{a, b}(p)=\frac{1}{a}\left(\begin{array}{cc}
k a & -p a b \\
p & -k b
\end{array}\right)
$$

see [16]. The monodromy matrix $L_{d, a}(p) L_{c, d}(q) L_{b, c}(p) L_{a, b}(r)$ yields one integral.

We perform the following reduction: in variables $x=a / c, y=b / d$ the mapping is expressed

$$
(x, y) \mapsto\left(\frac{p(q y+r x)-q(q x y+r)}{p(q x+r y)-q(r x y+q)} \frac{1}{x}, \frac{p(r x+q y)-r(q+r x y)}{p(q x+r y)-r(q x y+r)} \frac{1}{y}\right),
$$

and its integral is

$$
p^{2}\left(\frac{x}{y}+\frac{y}{x}\right)-p(r+q)\left(x+y+\frac{1}{x}+\frac{1}{y}\right)+\left(x y+\frac{1}{x y}\right) q r .
$$

In the next example we need four lattice parameters to ensure that the two lattice parameters on each quadrilateral differ. 


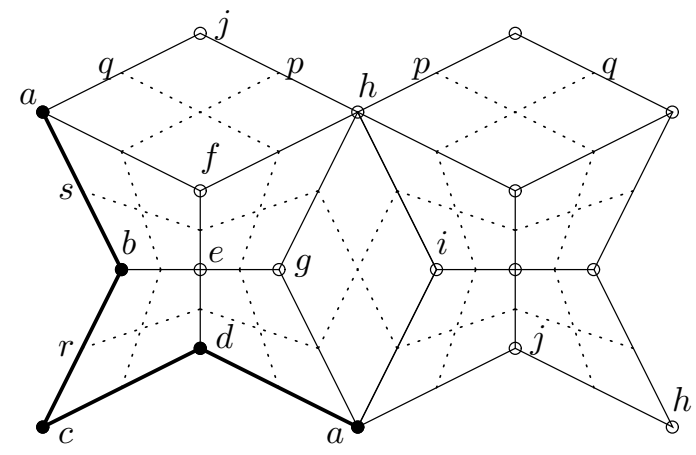

Figure 21

Given initial values $a, b, c, d$, the values $e, f, g, h, i$ can be determined by the following equations:

$$
\begin{aligned}
& Q_{r p}(b, c, e, d)=0, \\
& Q_{s p}(a, b, f, e)=0, \\
& Q_{r q}(e, d, g, a)=0, \\
& Q_{s q}(f, e, h, g)=0, \\
& Q_{r s}(g, a, h, i)=0, \\
& Q_{q p}(a, f, j, h)=0 .
\end{aligned}
$$

The values $a, b, c, d$ are repeated periodically on the 'staircase' which extends along a 'diagonal' of the quad-graph. The particular way of choosing lattice parameters ( $q, p, p, q$, see Figure 21, and we also take $r, s, s, r$ vertically) ensures that the periodic solution has the same period as the initial values. It is important to notice that when going one step to the right (on the $\mathbb{Z}^{2}$ part of the quad-graph) the lattice parameters $p, r$ are interchanged with $q, s$ respectively. Therefore, we consider the mapping

$$
(a, b, c, d, p, q, r, s) \mapsto(h, i, a, j, q, p, s, r) .
$$

After the transformation $x=a / c, y=b / d$ we are left with

$$
(x, y, p, q, r, s) \mapsto\left(\frac{1}{x} \frac{x_{n}}{x_{d}}, \frac{1}{y} \frac{y_{n}}{y_{d}}, q, p, s, r\right),
$$

with

$$
\begin{aligned}
x_{n}= & -q s p^{2}+p q(q s+p r) x+2 y p s r q-(r q+p s)(q s+p r) y x-y^{2} s r^{2} q \\
& -p q^{2} x^{2} r+s r(q s+p r) y^{2} x+\left(p^{2} q^{2}+s^{2} r^{2}\right) y x^{2}-y^{2} p s^{2} x^{2} r \\
x_{d}= & y^{2} r q p^{2} x-y^{2} r q^{2} p-y^{2} q s x^{2} p^{2}+y^{2} q^{2} s x p+y r^{2} s^{2}-y p q r^{2} x+2 y r q s x^{2} p \\
& -y p^{2} s x r-y q^{2} s x r+y q^{2} p^{2}-y p s^{2} x q-r^{2} q s x^{2}+r^{2} s p x+r s^{2} x q-p s^{2} r, \\
y_{n}= & \left(s^{2} p-s(q s+p r) x-s^{2} r y+\left(p r q+s^{3}\right) y x+x^{2} r q s-y q s x^{2} p\right) \\
& \cdot\left(-q s p+q(q s+p r) x+y s r q-\left(s r p+q^{3}\right) y x-q^{2} x^{2} r+x^{2} q^{2} p y\right), \\
y_{d}= & \left(-q s p+\left(p r q+s^{3}\right) x+y s r q-s(q s+p r) y x-x^{2} s^{2} r+x^{2} s^{2} p y\right) \\
& \cdot\left(q^{2} p-\left(\operatorname{srp}+q^{3}\right) x-y q^{2} r+q(q s+p r) y x+x^{2} r q s-y q s x^{2} p\right) .
\end{aligned}
$$

The monodromy matrix,

$$
L_{d, a}(q) L_{c, d}(p) L_{b, c}(r) L_{a, b}(s)
$$

yields one invariant, in reduced variables,

$$
x\left(1+y^{2}\right)(q s+p r)+y\left(x^{2}+1\right)(p q+s r)-\left(x^{2}+y^{2}\right) r q-\left(x^{2} y^{2}+1\right) p s .
$$


The linear part of the mapping is easily solved by

$$
p=c_{1}+c_{2}(-1)^{n}, q=c_{1}-c_{2}(-1)^{n}, r=c_{3}+c_{4}(-1)^{n}, s=c_{3}-c_{4}(-1)^{n}
$$

where $n$ is an integer counting the iterations of the map. Thus we may obtain an alternating two-dimensional map with one integral, cf. [28]. However, this map would take too much space to write explicitly. For special values of the parameters and one of the initial values we have observed that the growth of this map is quadratic, which indicates it is integrable.

\section{Multivaluedness of iterates of correspondences}

We have seen two examples in which correspondences arose: $(n, 0)$-reductions of the QD-system in section 3.2.1 and of the pKdV-equation in section 4.3.1. In general the multi-valuedness of the iterates of a correspondence would grow exponentially. However, for integrable correspondences one expects the multivaluedness to grow polynomially instead.

\subsection{The pKdV (3,0)-correspondence}

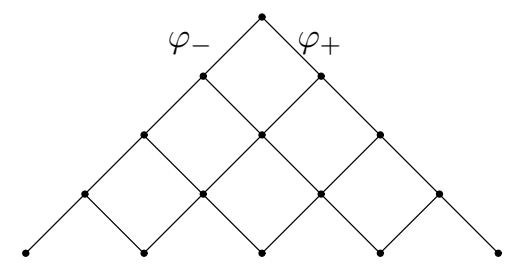

Figure 22: Polynomial growth of multivaluedness of iterates.
Generically the multi-valuedness of the $l$ th iterate of a two-valued map would be $2^{l}$. This is not the case here. Due to the $x \leftrightarrow y$ symmetry of the system (40) the correspondence $\varphi$ equals its own inverse. The relations $\varphi_{ \pm}^{-1}=\varphi_{\mp}$ imply that the $l$ th iterate of the correspondence is $(l+1)$-valued, see Figure 22 . All points on the same vertical line have the same value.

\subsection{The QD $(n, 0)$-correspondence}

As one can easily verify the mappings $\tau, \sigma$, given by equations $(20,21)$, satisfy

$$
\tau \sigma \tau=\sigma \sigma \sigma \quad \text { and } \quad \tau \sigma \sigma=\sigma \sigma \tau .
$$

Due to these relations the $l$ th iterate of the correspondence is $2 l$ valued, see figure 23 .

Proposition 4 With compositions of mappings (42), the lth iterate of the correspondence $(\sigma, \tau)$ is $2 l$ valued.

Proof: The relations (42) can be used to rewrite every word in two symbols $\sigma$ and $\tau$ of length $l$ into either a word that does not contain $\tau \sigma$ or a word that 


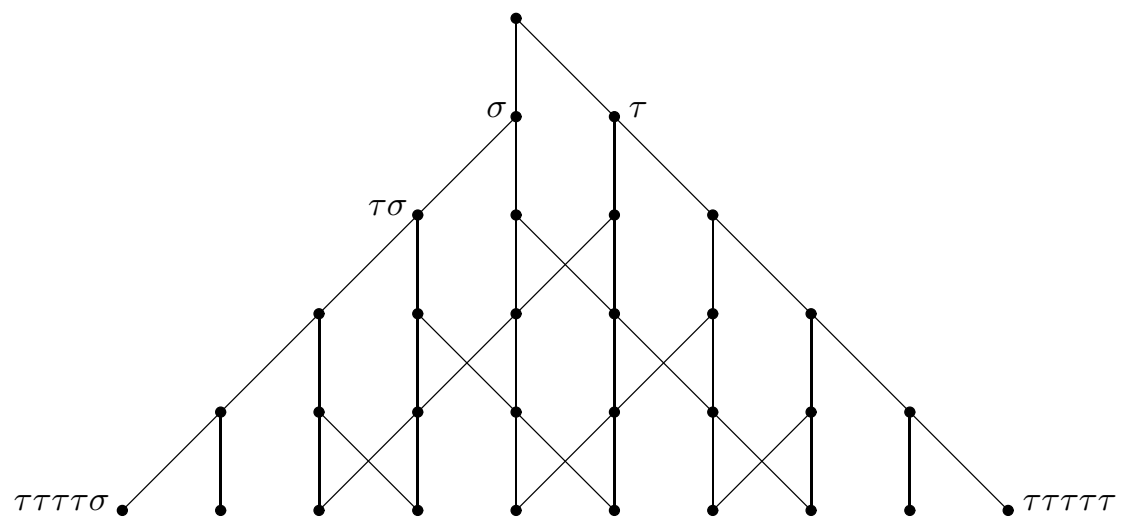

Figure 23: Graphical representation of Proposition 4

only contains $\tau \sigma$ at the end. There are $2 l$ such words:

$$
\begin{aligned}
& \tau \tau \tau \cdots \tau \tau \tau, \sigma \tau \tau \cdots \tau \tau \tau, \sigma \sigma \tau \cdots \tau \tau \tau, \ldots, \sigma \sigma \sigma \cdots \sigma \sigma \tau \\
& \tau \tau \tau \cdots \tau \tau \sigma, \sigma \tau \tau \cdots \tau \tau \sigma, \sigma \sigma \tau \cdots \tau \tau \sigma, \ldots, \sigma \sigma \sigma \cdots \sigma \sigma \sigma
\end{aligned}
$$

So there are at most $2 l$ inequivalent words of length $l$. To show that there are exactly $2 l$ inequivalent words of length $l$ we proceed by induction. We suppose there are are exactly $2 l$ inequivalent words of length $l$. For any three words $u, v, w$ we have $u v=u w \Rightarrow v=w$. So if two different words of length $l$ extend to an equivalent word of length $l+1$, this word can be written (we concatenate from the left) as $u=\tau v=\sigma w$. If a third word of length $l$ would extend to $u$ then we have $u=\tau z$ or $u=\sigma z$ which would imply $z=v$ or $z=w$ respectively. Therefore words of length $l$ that extend to words of length $l+1$ coincide at most pairwise, giving an lower bound of $2 l$ on the number of words of length $l+1$. However, since there are two words of length $l+1$ that are not equivalent to any other word, namely $\tau \tau \tau \cdots \tau \tau \tau$ and $\sigma \tau \tau \cdots \tau \tau \tau$, there are at least $2 l+2$ inequivalent words of length $l+1$.

The same relations hold in the $2 n$-dimensional case.

Proposition 5 The $2 n$-dimensional mappings $\tau=\tau_{2 n}$ (17) and $\sigma=\sigma_{2 n}$ (13) satisfy the relations (42).

Proof: That the second of the relations (42) holds is easily established as $\sigma \sigma$, i.e. the mapping $x_{k} \mapsto x_{k+2}$, clearly commutes with $\tau$.

For the first relation we solve the equations (12) for the $x_{i}$ in terms of the $\widehat{x_{k}}$ to find the inverse $\tau^{-1}$, with $i=1,2, \ldots, n$,

$$
\begin{aligned}
x_{2 i-1} & \mapsto x_{2 i-1} \frac{W_{2 i}^{n}}{W_{2 i-2}^{n}}, \\
x_{2 i} & \mapsto x_{2 i-2} \frac{W_{2 i-4}^{n}}{W_{2 i-2}^{n}},
\end{aligned}
$$


where

$$
W_{k}^{n}=\sum_{i=1}^{n-1}\left(\prod_{j=1}^{i-1} x_{2 j-1+k}\right)\left(\prod_{j=i}^{n-1} x_{2 j+2+k}\right) .
$$

One can verify that $\sigma\left(W_{k}^{n}\right)=Q_{k}^{n}$. This implies that $\sigma \tau^{-1}$ is given by

$$
\begin{aligned}
x_{2 i-1} & \mapsto x_{2 i+2} \frac{Q_{2 i}^{n}}{Q_{2 i-2}^{n}} \\
x_{2 i} & \mapsto x_{2 i-3} \frac{Q_{2 i-4}^{n}}{Q_{2 i-2}^{n}}, \quad i=1, \ldots, n
\end{aligned}
$$

which equals its inverse $\tau \sigma^{-1}$. Multiplying $\tau \sigma^{-1}=\sigma \tau^{-1}$ from the left by $\tau \sigma$ and from the right by $\sigma$, using $\tau \sigma \sigma=\sigma \sigma \tau$, gives us $\tau \sigma \tau=\sigma \sigma \sigma$.

Remark: One can also directly prove that the $\tau^{-1}$ provided is the inverse of $\tau$. This relies on the fact that $\tau\left(W_{k}^{n}\right)=Q_{k}^{n}$, which in terms of $Q$ amounts to the identity

$$
\prod_{i=1}^{n-1} Q_{2 i}^{n}=\sum_{i=1}^{n}\left(\prod_{j=1}^{i-1} x_{2 j-1}\right)\left(\prod_{j=i}^{n-1} x_{2 j+4}\right)\left(\prod_{j=1}^{n-2} Q_{2 j+2 i}^{n}\right)
$$

which can be proved using

$$
Q_{0}^{n-m} Q_{2}^{n}=x_{1} Q_{2}^{n-m-1} Q_{0}^{n}+Q_{2(n-m)}^{n}\left(\prod_{i=3}^{n-m+1} x_{2 i}\right)
$$

which in turn relies on a generalization of the relations (16),

$$
Q_{0}^{n-m}=Q_{2 m}^{n-m}\left(\prod_{i=2}^{m+1} x_{2 i}\right)+\left(Q_{0}^{m} \prod_{i=m}^{n-1} x_{2 i+1}\right) .
$$

\section{Concluding remarks}

We have shown that the staircase method provides integrals for mappings and correspondences derived as s-periodic reductions of lattice equations and systems of lattice equations. We also showed that such mappings and correspondences can be order-reduced systematically, using symmetries of the lattice equations. In all examples encountered the staircase method yields sufficiently many functionally independent integrals for the $d$-reduced mappings and correspondences to be completely integrable. However, we know the above statement is not true in general. In [34] periodic reductions of systems of P $\Delta$ Es are considered for which the staircase method does not provide sufficiently many integrals. However, in those cases it was observed that a certain linear combination of integrals factorises into a product of 2-integrals, from which then another integral can be obtained. 
For posing initial value problems we used the method laid out in [38]. Although, for the Boussinesq system we presented an alternative approach. We have calculated integrals for mappings/correspondences with dimension up to say 20. Therefore we were able to check functional independence of the integrals using the symbolic software package Maple [18].

Closed form expressions for integrals of mappings with arbitrary dimensions (corresponding to reductions with $s_{2}=-1$ ) have been given in [40,36]. There, the lattice equations are of Adler-Bobenko-Suris type $[2,3]$ and the integrals are expressed in terms of multisums of products. Their functional independence and involutivity is being investigated [37].

It is an open problem whether such closed form expressions can be obtained in general. In particular, we don't know whether such expressions can be given for the Bruschi-Calogero-Droghei equation, the Quotient-Difference algorithm and the Boussinesq system presented here, except for QD in the case $s_{1}=$ 0 . Another question is how to obtain symplectic structures for the mappings studied in this paper. This would enable one to conclude complete integrability.

We have obtained a few results on mappings of arbitrary dimension. These include an explicit $\left(2+(-1)^{n}\right)$-reduction of the mapping related to the $(n-1,1)$ reduction of the lattice potential KdV equation, as well as an explicit expression for a 2-integral of the mapping in the case that $n$ is even. We proved that the $(n-1,1)$-reduction of the Boussinesq system can be $d$-reduced with $d=6$ if 3 divides $n$ and with $d=2$ otherwise. Also, we presented two 3-integrals for this mapping (expressed in terms of the original variables). For the $(n-1,-1)$ reduction of the Boussinesq system we have showed that the mapping can be $d$ reduced with $d=6$ if 3 divides $n+1$ and with $d=2$ otherwise. Finally, the $(n, 0)$ reduction of the QD-system yields a $2 n$-dimensional 2-valued correspondence. We have given an explicit expression for this correspondence and showed that its $n$th iterate is $2 n$-valued. It would be interesting to investigate other ways of establishing such a result because one does not always have explicit expressions at hand.

\section{Acknowledgement.}

This research has been funded by the Australian Research Council through the Centre of Excellence for Mathematics and Statistics of Complex Systems. We are grateful for the hospitality of the Isaac Newton Institute during the 2009 program on Discrete Integrable Systems.

\section{References}

[1] M.J. Ablowitz and F.J. Ladik, A nonlinear difference scheme and inverse scattering, Stud. Appl. Math. 55 (1976) 213-229; On the solution of a class of nonlinear partial difference equations, ibid. 57 (1977) 1-12. 
[2] V.E. Adler, A.I. Bobenko, Yu.B. Suris, Classification of integrable equations on quad-graphs. The consistency approach, Commun. Math. Phys. 233 (2003), 513-543. arXiv:nlin/0202024.

[3] V.E. Adler, A.I. Bobenko, Yu.B. Suris, Discrete nonlinear hyperbolic equations. Classification of integrable cases, Funct. Anal. Appl. 43 (2009), 3-21. arXiv:nlin/0705.1663

[4] V.E. Adler and A.P. Veselov, Cauchy Problem for Integrable Discrete Equations on Quad-Graphs Acta Applicandae Mathematicae 84: 237-262, 2004.

[5] C. Brezinski, Padé-type approximation and general orthogonal polynomials, International Series in Numerical Mathematics, Birkhauser, Basel, 1980.

[6] M. Bruschi, F. Calogero and R. Droghei, Tridiagonal matrices, orthogonal polynomials and Diophantine relations I, J. Phys. A: Math. Theor. 40 (2007), 9793-9817.

[7] M. Bruschi, O. Ragnisco, P. M. Santini, and T.G. Zhang, Integrable symplectic maps, Physica D 49 (1991), 273-294.

[8] J.H. McCabe, The quotient difference algorithm and the Padé table: An alternative form and a general continued fraction, Mathematics of Computation 41(6) (1983), 183-197.

[9] F. Calogero and M. C. Nucci, Lax pairs galore, J. Math. Phys. 32 (1991) $72-74$.

[10] H.W. Capel, F.W. Nijhoff and V.G. Papageorgiou, Complete Integrability of Lagrangian Mappings and Lattices of KdV Type, Physics Letters 155A (1991), 377-387.

[11] E. Date, M. Jimbo and T. Miwa, Method for Generating Discrete Soliton Equations I-V, J. Phys. Soc. Japan 51 (1982), 4116-4124, 4125-4131, 52 (1983), 388-393, 761-765, 766-771.

[12] B. Fuchssteiner, Coupling of completely integrable system: the perturbation bundle. In: P.A. Clarkson, Editor, Applications of analytic and geometric methods to nonlinear differential equations, Kluwer, Dordrecht (1993) 125-138.

[13] F. Haggar, G.B. Byrnes, G.R.W. Quispel and H.W. Capel, $k$-integrals and $k$-Lie symmetries in discrete dynamical systems, Physica 233A (1996) 379394.

[14] M. Hay, Discrete Lax pairs, Reductions and Hierarchies, PhD thesis, The University of Sydney (2008).

[15] P. Henrici and Bruce O. Watkins, Finding zeros of a polynomial by the Q-D algorithm, Communications of the ACM 8(9) (1965), 570-574. 
[16] W. Hereman, Symbolic computation of Lax Pairs of integrable nonlinear difference equations on quad-graphs, http://www.newton.ac.uk/programmes/DIS/seminars/062914001.pdf.

[17] R. Hirota, Nonlinear Partial Difference Equations I-III, J. Phys. Soc. Japan 43 (1977) 1424-1433, 2074-2078, 2079-2089.

[18] Maple, http://www.maplesoft.com/products/Maple/index.aspx

[19] A. Nagai, T. Tokihiro and J. Satsuma, The Toda Molecule Equation and the $\epsilon$-Algorithm, Mathematics of Computation 67 (1998) 1565-1575

[20] F.W. Nijhoff and H.W. Capel, The Discrete Korteweg-de Vries equation, Acta Applicandae Mathematicae 39 (1995) 133-158.

[21] F.W. Nijhoff, V.G. Papageorgiou and H.W. Capel, Integrable TimeDiscrete Systems: Lattices and Mappings, Ed. P.P. Kulish, in: Quantum Groups, Lecture Notes in Mathematics vol. 1510, pp. 312-325, Berlin/New York, Springer Verlag, 1992.

[22] F.W. Nijhoff, V.G. Papageorgiou, H.W. Capel and G.R.W. Quispel, The Lattice Gel'fand-Dikii Hierarchy, Inverse Problems 8 (1992) 597-621.

[23] F.W. Nijhoff, G.R.W. Quispel and H.W. Capel, Direct linearization of nonlinear difference-difference equations, Phys. Lett., 97A (1983) 125-128.

[24] F.W. Nijhoff and A.J. Walker, The discrete and continuous Painlevé VI hierarchy and the Garnier systems, Glasgow Math. J. 43A (2001), 109123.

[25] V. Papageorgiou, B. Grammaticos and A. Ramani, Orthogonal polynomial approach to discrete Lax pairs for initial boundary-value problems of the QD algorithm, Letters in Mathematical Physics 34(2) (1995), 91-101.

[26] V.G. Papageorgiou and F.W. Nijhoff, On some Integrable Discrete-Time Systems associated with the Bogoyavlensky Lattices, In: Statistical Mechanics, Soliton Theory and Nonlinear Dynamics, Festschrift in honour of H.W. Capel's 60th Birthday, eds. G.R.W. Quispel, F.W. Nijhoff and J.H.H. Perk, Physica 228A (1996), 172-188.

[27] V.G. Papageorgiou, F.W. Nijhoff, and H.W. Capel, Integrable mappings and nonlinear integrable lattice equations, Phys. Lett. A 147 (1990) 106114 .

[28] G.R.W. Quispel, An alternating integrable map whose square is the QRT map, Phys. Lett. A 307 (2003) 504.

[29] G.R.W. Quispel, H.W. Capel, V.G. Papageorgiou and F.W. Nijhoff, Integrable mappings derived from soliton equations, Physica A 173 (1991) 243-266. 
[30] G.R.W. Quispel, F.W. Nijhoff, H.W. Capel and J. van der Linden, Linear Integral Equations and Nonlinear Difference-Difference Equations, Physica 125A (1984) 344-380.

[31] G.R.W. Quispel, J.A.G. Roberts and C.J. Thompson, Integrable Mappings and Soliton Equations I, Phys. Lett. 126A (1988) 419-421.

[32] G.R.W. Quispel, J.A.G. Roberts and C.J. Thompson, Integrable Mappings and Soliton Equations II, Physica 34D (1989) 183-192.

[33] O. Rojas, P.H. van der Kamp and G.R.W. Quispel, Lax representations for integrable mappings, In preparation.

[34] P.E. Spicer, F.W. Nijhoff, P.H. van der Kamp, Higher analogues of the discrete-time Toda equation and the quotient-difference algorithm (2010) arXiv:nlin/1005.0482.

[35] A.S. Tongas and F.W. Nijhoff, The Boussinesq integrable system. Compatible lattice and continuum structures, Glasgow Math. J. 47A (2005) 205-219. arXiv:nlin/0402053

[36] D. Tran, P.H. van der Kamp, G.R.W. Quispel, Closed-form expressions for integrals of traveling wave reductions of integrable lattice equations, J. Phys. A: Math. Theor. 42 (2009) 225201.

[37] D. Tran, P.H. van der Kamp, G.R.W. Quispel, Involutivity of sine-Gordon, modified and potential KdV maps (2010) arXiv:nonl/1010.3471.

[38] P.H. van der Kamp, Initial value problems for lattice equations, J. Phys. A: Math. Theor. 42 (2009) 404019.

[39] P.H. van der Kamp, Growth of degrees of integrable mappings, Journal of Difference Equations and Applications (2010) arXiv:nonl/1005.2065v1.

[40] P.H. van der Kamp, O. Rojas and G.R.W. Quispel, Closed-form expressions for integrals of mKdV and sine-Gordon maps, J. Phys A: Math Gen. 40 (2007) 1278912798.

[41] A.P. Veselov, Growth of the number of images of a point under iterates of a multivalued map, Math. Notes 49 (1991), 134-139.

[42] A.P. Veselov, Integrable maps, Russian Mathematical Surveys 46 (1991), $1-51$. 